\title{
Harmonizing Prognostics and Health Management with Risk Analysis for Smart Manufacturing Systems
}

\author{
A Graduate Thesis \\ Presented to the Department of Systems \& Information Engineering \\ University of Virginia $•$ Charlottesville, Virginia \\ In Partial Fulfillment of the Requirements for the Degree \\ Master of Science in Engineering
}

\author{
Author \\ Michael L. Malinowski \\ May 2016
}

Thesis Committee

Dr. Yacov Haimes (Thesis Advisor, SIE UVa)

Dr. Peter Beling (Thesis Co-Advisor, SIE UVa)

Dr. Barry Horowitz (Committee Chair, SIE UVa)

Dr. James Lambert (Committee Member, SIE UVa)

Dr. Stephen Adams (Committee Member, SIE UVa) 


\begin{abstract}
This thesis develops a methodology for the design phase of a Prognostics Health Management (PHM) system of a manufacturing process. The methodology (i) builds upon Hierarchical Holographic Modeling (HHM), Risk Filtering, Ranking, and Management (RFRM), and Fault Tree Analysis (FTA); (ii) provides scope and direction for a PHM system by identifying a prioritized set of targets that would most benefit from PHM capabilities; and (iii) tests the outcome of the developed methodology with two case studies with U.S. manufacturing companies. Currently, there are multiple methods to determine the major failure modes of a system following an accident or catastrophe. However, the proposed methodology in this thesis allows for a thorough analysis to be conducted even before a failure occurs in a manufacturing environment. Another important goal of this thesis is to demonstrate the compatibility and synergy between two seemingly different methodologies/processes: Risk analysis and PHM for manufacturing. More specifically, this research demonstrates that the theory, methodology, and current practice of the system-based risk analysis are harmonious and compatible with PHM. The compatibility between Risk Analysis and PHM is further solidified through an investigation and comparison between the Dynamic Roadmap for Risk Modeling, Planning, Assessment, Management, and Communication with the Major Design Components of Prognostics and Health Management. The major benefits to PHM from integrating Risk Analysis methodology are revealed through the two case studies in U.S. manufacturing industry.
\end{abstract}




\section{Acknowledgements}

I would like to thank everyone who supported me throughout my journey through graduate school at the University of Virginia. Professor Haimes, thank you so much for being more than just a research advisor, but also a teacher, mentor, and friend. You always had my back and reminded me to enjoy my time as a student. Professor Beling, I would like to thank you for all your advice and guidance which truly helped shape my research. I would also like to thank the rest of my committee, Professor Horowitz, Professor Lambert, and Dr. Stephen Adams, for helping to ensure my successful thesis.

I would like to say thank you to our research supporters at the National Institute of Standards and Technology, especially Brian Weiss and Jeremy Marvel. I must also thank the graduate students on my research team, Jerry Heddy and Ben Choo, for struggling with me and supporting me through research trips and deadlines.

I would like to thank my family for their continuous love and support: my parents, Robert and Sylvia Malinowski, my younger siblings, Kristen and David, and all of my cousins and external family. I am also grateful to my girlfriend Emily Richards for pushing me to finish my work on time; I can't wait to walk with you in May.

Thank you to all the students, teachers, and friends I made along the way. I have learned so much from all of you here in Charlottesville and I wish you all the best. 


\section{Table of Contents}

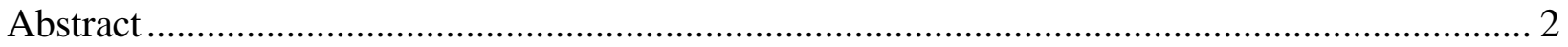

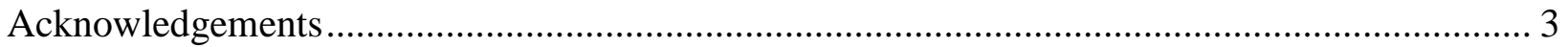

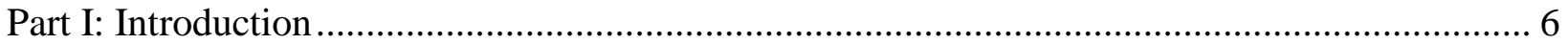

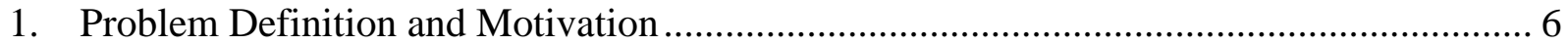

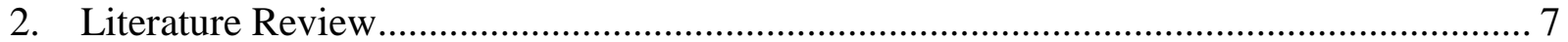

3. Smart Manufacturing System of Systems ................................................................... 10

Part II: The Compatibility of Risk Analysis and Prognostics and Health Management .............. 12

Part III: Case Studies with Smart Manufacturing Companies .................................................... 19

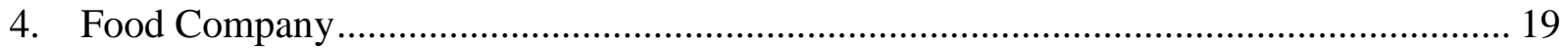

4.1 Food Company - Manufacturing Process Overview ……................................................ 19

4.2 Food Company - Objectives and Known Issues ............................................................. 22

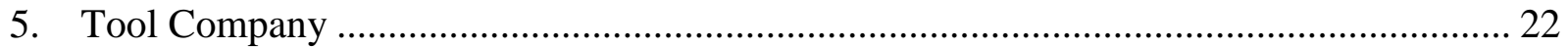

5.1 Tool Company - Manufacturing Process Overview....................................................... 22

5.2 Tool Company - Objectives and Known Issues .......................................................... 24

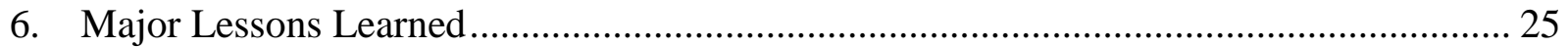

Part IV: Methodological Framework: Systems-Based Risk Analysis ......................................... 26

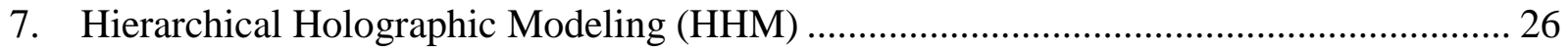

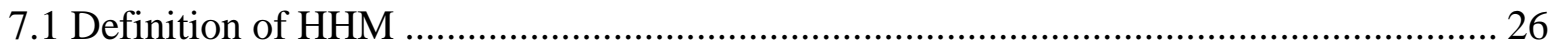

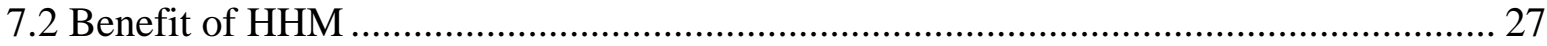

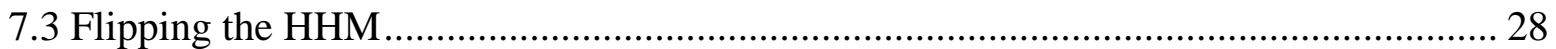

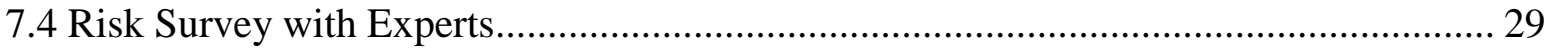

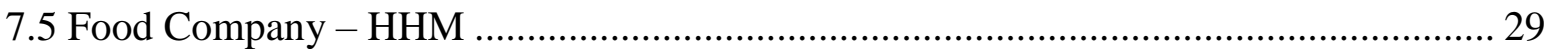

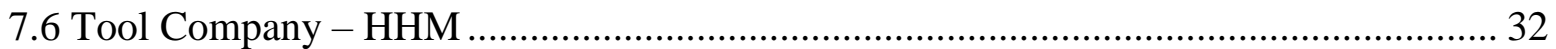

8. Risk Filtering, Ranking, and Management (RFRM) …………………………............ 35

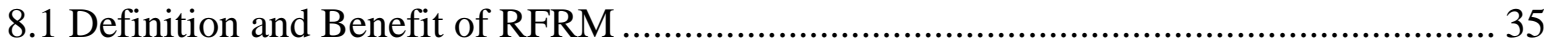

8.2 RFRM Phases 1 \& 2: Scenario Identification / Scenario Filtering .................................... 36

8.3 RFRM Phases 3 \& 4: Bi-Criteria Filtering / Quantitative Ranking .................................. 38

8.4 RFRM Phase 5: Multi-Criteria Evaluation .................................................................. 41

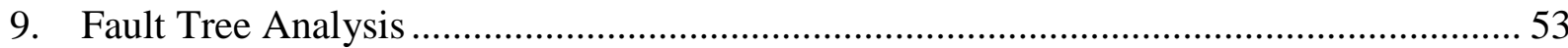

9.1 Food Company Fault Tree Analysis .......................................................................... 55

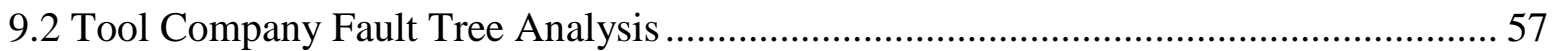

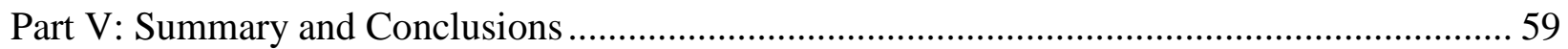

10. Major Findings and Contributions ………………................................................ 59 


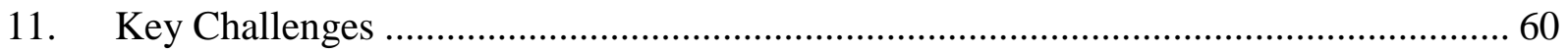

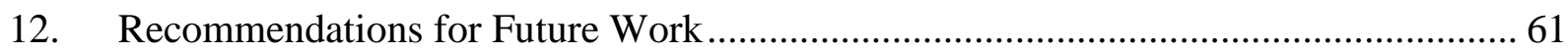

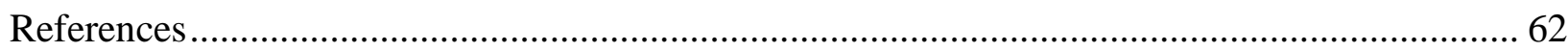

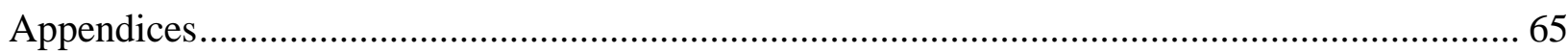

Appendix A. Risk in Manufacturing Processes Survey ................................................... 65

Appendix B. Food Company - Full List of Risk Scenarios .............................................. 68

Appendix C. Tool Company - Full List of Risk Scenarios ............................................... 70

Appendix D. Support Flowcharts for RFRM Phase 5 Multi-Criteria Evaluation.................... 74 


\section{Part I: Introduction}

\section{Problem Definition and Motivation}

Manufacturing industries are expanding in both size and complexity. Many are undergoing major transformations and adapting new technologies (e.g., sensing, automation, machining, robotics) to provide competitive, high-quality products to their customers. As the complexity of manufacturing processes increases, there is a growing need to identify, characterize, and communicate metrics to decision makers regarding the health of the system. Specifically, managers are seeking to enhance their ability to gather timely and actionable intelligence about their system that can be used to achieve the following major objectives: minimize cost, minimize production/maintenance time, maximize system health, maximize product quality, and maximize product output.

Many systems and methodologies have been developed to provide this type of intelligence, especially within the field of Prognostics and Health Management (PHM). In particular, PHM seeks to provide tools for predicting remaining usable life (RUL) of equipment, for diagnosing errors or failures, and for developing maintenance or mitigation strategies. However, there are multiple challenges to implementing PHM across all manufacturing sectors. Manufacturing systems are inherently complex, and can involve multiple subsystems, processes, and components that are all reasonable targets for the development of PHM systems. This selection problem is made more difficult because the potential costs and benefits of those PHM systems are subject to random and known uncertainties. Thus, manufacturers require a way to determine which subsystems or components are most critical, and thus would most benefit from an established PHM system.

The field of Risk Analysis (modeling, assessment, management, and communication) provides systematic and well-established approaches to identifying the major risk scenarios that would impair a system from achieving its major objectives. However, the methodologies require modifications so that they can be applied to identify targets/components within a manufacturing process that would most benefit from a PHM system. This research seeks to establish this modified methodological approach so that it can be followed by any manufacturing company. Specifically, the methodology incorporates Hierarchical Holographic Modeling (HHM), Risk 
Filtering, Ranking, and Management (RFRM), and Fault Tree Analysis (FTA) to provide scope and direction for the PHM system designer.

The academic field of Risk Analysis provides many additional tools, resources, methodologies, and theories that could advance the growing field of Prognostics and Health Management. Therefore, an additional objective of this research is to merge these two schools of thought to promote additional collaboration. In particular, the research demonstrates that the current practice of the system-based Risk Analysis are harmonious and compatible with PHM, specifically in regard to its application of ensuring the "health" (safety and optimal operability and performance) of manufacturing system of systems. The similarities between Risk Analysis and PHM are solidified through an investigation and comparison between the "Guiding Principles" of Risk Analysis and the major phases of PHM design. The benefits to PHM from implementing Risk Analysis methodology are further outlined through multiple case studies in U.S. manufacturing industry.

\section{Literature Review}

Smart Manufacturing Systems require advanced technologies that facilitate widespread information flow within the system's components and subsystems. This information can include the health, performance, and risk of the system in failing to meet an objective (Jung, Morris, Lyons, Leong, \& Cho, 2015). The engineering focus of Prognostics and Health Management (PHM) is coupled with smart manufacturing. The term "prognostics" refers to the prediction of the future status, health, or performance of components and systems. The term "health management" on the other hand refers to the process of making maintenance and logistics decisions from the prognostics information, available resources, and operational demand (Barajas \& Srinivasa, 2008). The focus of health management is to minimize operational loss and to maximize the objectives established by the facility (Lee, Wu, Zhao, Ghaffari, Liao, \& Siegel, 2014).

The use of PHM models to improve manufacturing performance has been demonstrated in numerous case studies within automotive (Holland, Barajas, Salman, \& Zhang, 2010), aerospace (Batzel \& Swanson, 2009), machine tool (Biehl, Staufenbiel, Recknagel, Denkena, \& Bertram, 2012), and power generation (Hofmeister, Wagoner, \& Goodman, 2013) industries. However, as 
manufacturing processes increase in size and complexity, it can become exceedingly difficult to determine which components or subsystems can most benefit from a PHM system model. Even data-driven approaches, which rely on historical data and mathematical models, lose accuracy and become less predictive as complexity increases (Bai, Wang, \& Hu, 2015). When available resources for PHM efforts are limited, designers and implementers of PHM systems face a difficult problem in deciding where to deploy these scarce resources to maximize benefit. A smart manufacturing system may involve multiple subsystems or processes that present reasonable targets for the development of PHM systems (Barajas \& Srinivasa, 2008). This selection problem is made more difficult because the potential costs and benefits of those potential PHM systems are subject to random and known uncertainty (Feldman, Jazouli, \& Sandborn, 2009) (Hou-bo, \& Jian-min, 2011).

Numerous systems-based risk analysis methodologies designed to support decision-makers within manufacturing industries have successfully been developed and deployed (Lee, Lv, \& Hong, 2013) (Fernández, \& Pérez, 2015), including Hierarchical Holographic Modeling (HHM) (Haimes, 2009) and Risk Filtering, Ranking, and Management (RFRM) (Haimes, Kaplan, \& Lambert, 2002) (Haimes, 2009). The original purpose of these methods (within the field of risk analysis) was to identify the most critical sources of risks to a system and to provide risk assessment, risk management, and risk communication (Haimes, 2012). With a few modifications, the critical risks identified in the HHM and RFRM processes can be used to identify the most critical components and subsystems that would most benefit from a PHM system or model.

Additionally, there currently exist multiple methods that seek to determine the major failure modes of a system after an accident or catastrophe (Cocheteux, Voisin, Levrat, \& Iung, 2009) (Lee et al., 2014) (Vykydal, Plura, Halfarová, \& Klaput, 2015). The proposed methodology in this thesis would allow for a thorough analysis to be conducted even before a failure occurs in a manufacturing environment.

Hierarchical Holographic Modeling is defined as a holistic philosophy and methodology aimed at capturing and representing the essence of the inherent diverse characteristics and attributes of a system (Haimes, 2012). These system attributes include, but are not limited to, the multiple 
aspects, perspectives, facets, views, dimensions, and hierarchies. The mathematical and systems approach to holographic modeling reveals the interconnectedness, and the interdependencies among the system's objective functions, constraints, decision variables, and inputs/outputs (Haimes, 2009). The term holographic refers to the desire to have a multi-view image of a system (Crowther et al., 2004). For example, the risk to a system due to emergent forced changes (EFCs) can be represented from its multiple perspectives, which are related to time and geography, and include, but are not limited to: (1) economic, (2) health, (3) technical, (4) political, and (5) social perspectives. To capture a holographic outcome, the modeling team that performs the analysis must represent a broad array of experience and knowledge (Haimes, 2009).

HHM has been successfully utilized in numerous projects and for multiple agencies, including the President's Commission on Critical Infrastructure Protection (PCCIP), the Federal Bureau of Investigation (FBI), the National Aeronautics and Space Administration (NASA), the Virginia Department of Transportation (VDOT), and the U.S. Army National Ground Intelligence Center (NGIC) (Haimes, 2009) (Lambert, Haimes, Li, Schooff, \& Tulsiani, 2001). The PCCIP utilized HHM to determine the major hardware, software, human, and environmental risks to a supervisory control and data acquisition system (Chittester, \& Haimes, 2004). The FBI developed an HHM model to identify varying perspectives, motives, and weaknesses between homeland defenders and terrorist networks (Haimes, \& Horowitz, 2004). For VDOT, the HHM method identified major interdependencies within Virginia's transportation infrastructure and outlined critical sectors that were most sensitive to disruptions (Crowther, Dicdican, Leung, Lian, \& Williams, 2004). Finally for the Army NGIC, HHM was used prior to a major deployment to identify the critical state variables of the target host country, U.S. forces, and U.S. allies (Dombroski, Haimes, Lambert, Schlussel, \& Sulcoski, 2002).

In total risk management, it is necessary to identify, prioritize, assess, and manage potential risk scenarios to a large-scale system. Stakeholders and decision-makers must consider the likelihoods and consequences of each risk to produce acceptable mitigation options. The Risk Filtering, Ranking, and Management (RFRM) methodology offers eight major phases to guide total risk management in an HHM system (Haimes, 2002). The eight phases are: 1 - Scenario Identification, 2 - Scenario Filtering, 3 - Bi-criteria filtering, 4 - Multi-criteria Evaluation, 5 - 
Quantitative Ranking, 6 - Risk Management, 7 - Safeguarding Against Missing Critical Items, and 8 - Operational Feedback.

The guiding force behind RFRM is the identification of head topics, which represent major concepts or perspectives of success, and subtopics, which provide detailed requirements or sources of risk (Haimes, 2009). However, it is often impractical to evaluate hundreds of sources of risk when evaluating a large system. Therefore, the risk scenarios and sources should be filtered based on professional experience, expert knowledge, and statistical data. It is also important to consider a variety of risks such as those related to hardware, software, organizational failure, human error, budget, schedule slip, and performance criteria (Haimes, 2002).

The RFRM methodology has been successfully deployed on numerous systems for multiple agencies, including the NASA, the Federal Aviation Administration (FAA), the VDOT, the National Ground Intelligence Center (NGIC), and the Department of Homeland Security (DHS) (Haimes, 2009). NASA used RFRM to identify the most common risk scenarios facing future space missions (e.g., inadequate oversight teams), and to compare management strategies to mitigate those risks (e.g., restructure existing teams or hire external consultants) (Haimes, 2009). For VDOT, the RFRM method ranked and prioritized the potential shutdowns of various transportation infrastructure assets (e.g., roads, highways, or bridges) according to their impacts on state transportation inoperability and economic loss (Crowther et al., 2004). Finally, the Army NGIC used the RFRM method to identify the risk scenarios that allied forces might encounter in a foreign country that occurred with the highest likelihood probability and produced the most severe results (e.g., loss of life or major asset) (Dombroski et al., 2002).

\section{Smart Manufacturing System of Systems}

Smart manufacturing is the integration of information, technology, and human ingenuity to promote rapid revolution in manufacturing intelligence across all business sectors (Chand, \& Davis, 2010). In the context of an industrial facility, smart manufacturing incorporates the available data across all systems, machines, and components in order to facilitate improvements towards achieving the pre-defined objectives of the organization (e.g., cost, safety, production, or environmental sustainability). Manufacturing industries today are expanding in both size and 
complexity to promote smart manufacturing. Many are undergoing major transformations and adapting new technologies (e.g., sensing, automation, machining, robotics) to provide competitive, high-quality products to their customers. As the complexity of these manufacturing processes increases, so too must their system models expand. Most organizational and technology-based manufacturing systems are hierarchical in nature (Alvandi, Bienert, Li, \& Kara, 2015) (He, Zhang, \& Li, 2014), so their models must reflect this reality. In the context of smart manufacturing, the natural structure of these systems of systems can be represented as follows:

1. System (e.g., factory)

a. Subsystem (e.g., work station)

i. Machine (e.g., lathe)

1. Component (e.g., spindle)

a. Primitive (e.g., cutting insert)

Smart Manufacturing Systems require advanced technologies that facilitate widespread information flow within the system's components and subsystems. This information can include the health, performance, and risk of the system in failing to meet an objective (Jung, Morris, Lyons, Leong, \& Cho, 2015). The engineering focus of Prognostics and Health Management (PHM) is coupled with smart manufacturing. The term "prognostics" refers to the prediction of the future status, health, or performance of components and systems. A commonly used metric within engineering prognostics is the remaining usable life (RUL) of a machine or system (National Institute of Standards and Technology, 2014). The term "health management" on the other hand refers to the process of making maintenance and logistics decisions from the prognostics information, available resources, and operational demand (Barajas \& Srinivasa, 2008). The focus of health management is to minimize operational loss and to maximize the objectives established by the facility (Lee, Wu, Zhao, Ghaffari, Liao, \& Siegel, 2014). 


\section{Part II: The Compatibility of Risk Analysis and Prognostics and Health Management}

A major objective of this thesis is to merge the field of Risk Analysis (Modeling, Planning, Assessment, Management, and Communication) with Prognostics and Health Management (PHM). Essentially, I shall demonstrate that the theory, methodology, and current practice of the system-based risk analysis are harmonious and compatible with PHM, specifically in regard to its application of ensuring the "health" (safety and optimal operability and performance) of manufacturing system of systems. Additionally, I will show that the methodologies for precursors to risk analysis are just as valid and applicable to manufacturing and PHM. The similarities between risk analysis and PHM shall be solidified through an investigation and comparison between the Dynamic Roadmap of Risk Analysis and the major phases of PHM design. The benefits to PHM from implementing Risk Analysis methodology shall be outlined through multiple case studies in U.S. manufacturing industry.

The dynamic roadmap for Risk Modeling, Planning, Assessment, Management, and Communication for systems of systems can be broken down into four major parts: (1) The Evolving Base, (2) The Journey, (3) The Compass - Guiding Principles, and (4) The Interim Destination and Back to Part One. A visual diagram of the roadmap can be seen in Figure 1 (Haimes, 2012). 


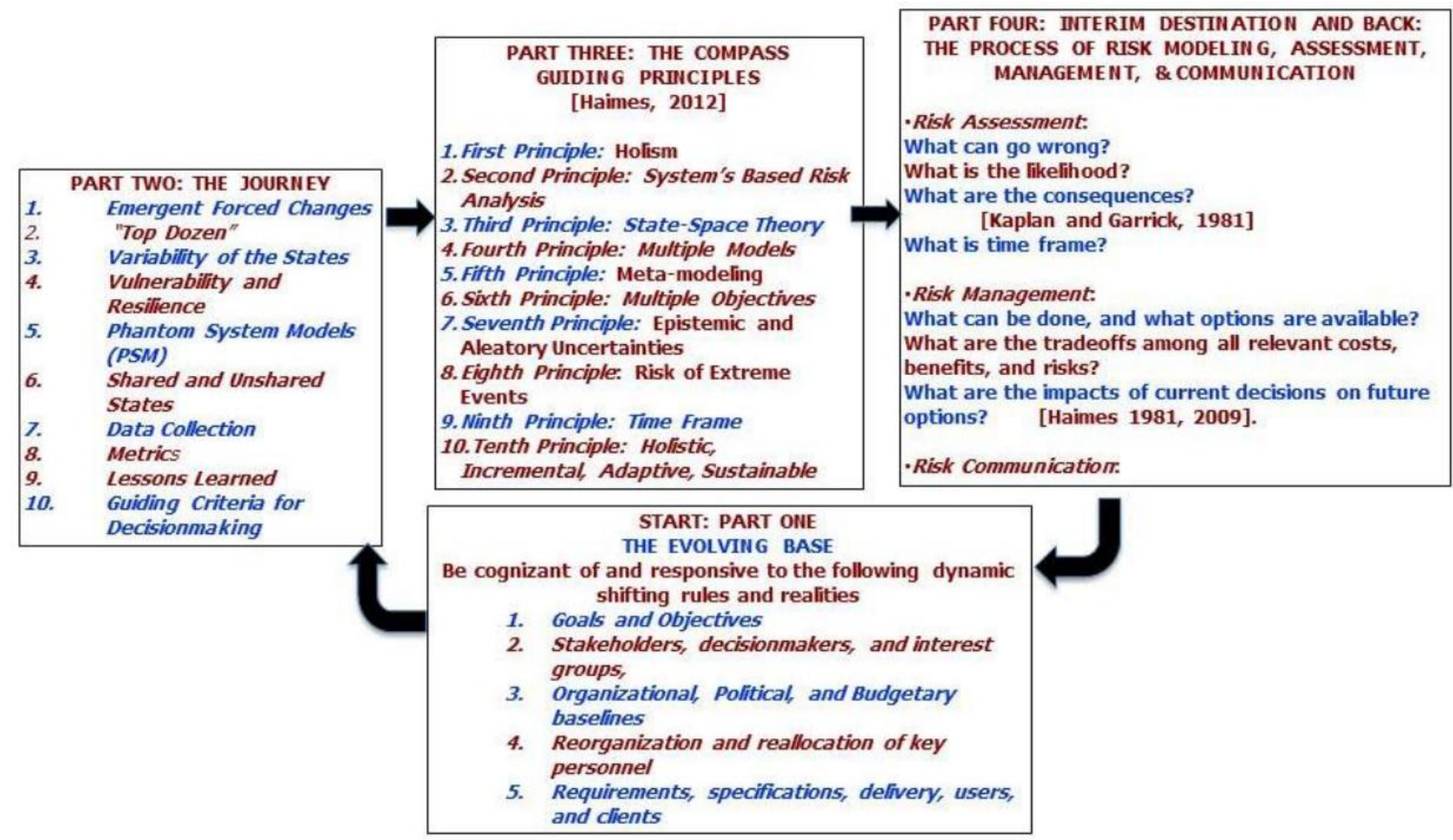

Figure 1. Dynamic Roadmap for Risk Modeling, Planning, Assessment, Management, and Communication for Systems of Systems.

The first part of the risk analysis roadmap is called "The Evolving Base", and it refers to a number of shifting rules and realities that risk analysts and decision makers must always keep in mind. Among these items are the goals, stakeholders, restrictions, personnel, requirements, and clients of the system. Since the evolving base is always changing, so too must the risk models, analysis, and management decisions change to reflect the base (Haimes, 2012). The field of PHM also addresses this reality. In fact, in the Measurement Science Roadmap for PHM of Smart Manufacturing Systems (SMS) it is stated that "as equipment, processes and operating environments evolve, failure modes and physics can change. PHM systems need to be open and adaptive to changes in all these areas" (National Institute of Standards and Technology, 2015). It is clear that both schools of thought embrace the idea of an evolving base, and the need to constantly adapt in order to remain relevant and true to the system.

The second part of the dynamic roadmap is called "The Journey", and it encompasses 10 major guidelines for any complete risk analysis study: (1) Emergent Forced Changes, (2) Top Dozen, (3) Variability of the States, (4) Vulnerability and Resilience, (5) Phantom System Models 
(PSM), (6) Shared and Unshared States, (7) Data Collection, (8) Metrics, (9), Lessons Learned, and (10) Guiding Criteria for Decision Making. Let's compare these risk guidelines to the four major design components of Prognostics and Health Management: (1) PHM Design, (2) Sensing and Feature Analysis, (3) Anomaly Detection \& Diagnostics, and (4) Response. Figure 2 shows a visual diagram of the PHM design components (NIST, 2015). A visual mapping of the alignment between these two fundamental aspects of Risk Analysis and PHM can be seen in Figure 3.

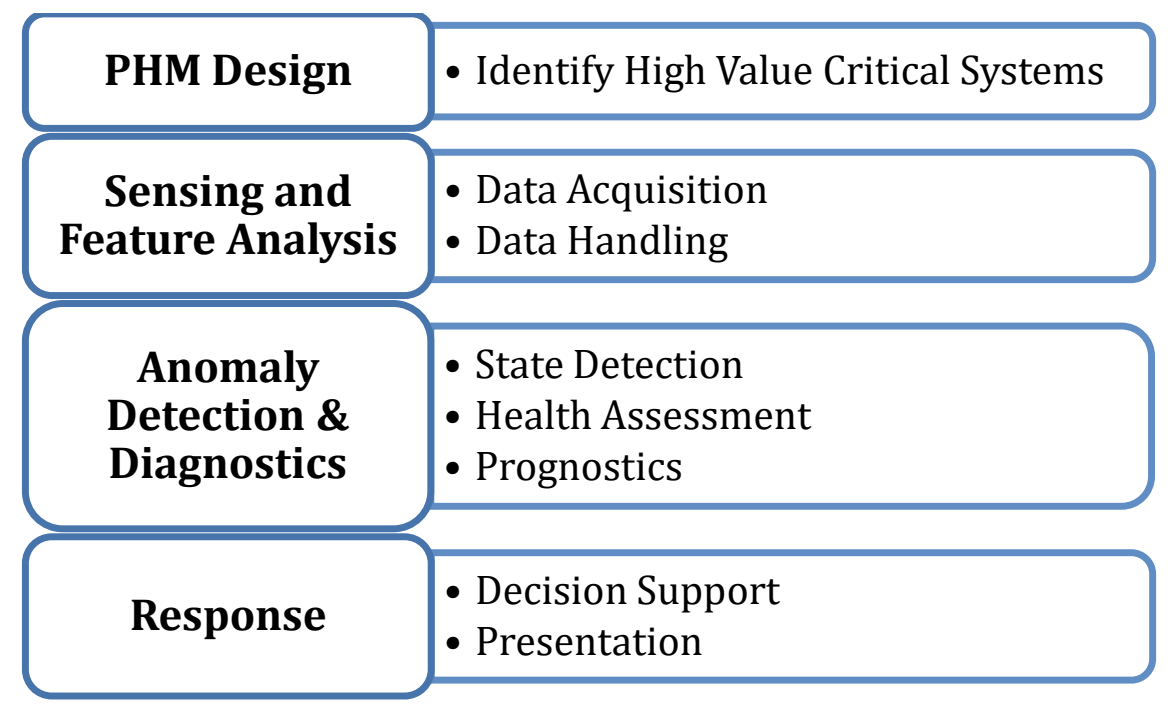

Figure 2. Major Design Components of Prognostics and Health Management.

The first two guidelines of the risk analysis journey are referred to as Emergent Forced Changes and the Top Dozen. They state the need to embrace the modified theory of scenario structuring (Kaplan \& Garrick, 1981) (Haimes, 2009), identify the most pertinent risk scenarios to the system of interest, and then finally to reduce the list to a manageable number. Risk analysis offers multiple methodologies towards accomplishing this task, including Hierarchical Holographic Modeling (HHM) and Risk Filtering, Ranking, and Management (RFRM). The field of PHM also embraces this need. In fact, the first major design component titled PHM Design labels the first step as to "identify high value critical systems." Unfortunately, a smart manufacturing system of systems may involve multiple subsystems or processes that present reasonable targets for the development of PHM systems (Barajas \& Srinivasa, 2008). This selection problem is made even more difficult because the potential costs and benefits of those potential PHM systems are subject to uncertainties (Feldman, Jazouli, \& Sandborn, 2009) (Hou- 
bo, \& Jian-min, 2011). Fortunately, the holistic and systematic risk methodologies can help identify these critical subsystems of interest for PHM.

The third, fourth, fifth, and sixth guidelines of risk analysis are Variability of the States, Vulnerability and Resilience, Phantom System Models, and Shared and Unshared States. These guidelines recommend first identifying a list of the shared and unshared states (e.g., decision variables, inputs, outputs, and objectives) in order to recognize the interconnectedness and interdependencies between subsystems and components. Next, the risk analyst must account for variability amongst the states over time, and try to define the most appropriate time frame. Finally, the vulnerability and resilience of each subsystem must be assessed with respect to the previously defined risk scenarios. PHM also embraces the need to identify the critical states of the system, as well as the variability of those states. Within the third PHM design component titled Anomaly Detection \& Diagnostics, State Detection is listed as the very first step. This phase of PHM also describes the need to identify any precursors to equipment failure, and finally to estimate remaining usable life (RUL) of the system. The metric RUL is essentially the PHM version of vulnerability and resilience defined in the risk analysis guidelines. The only difference is that RUL provides a narrower, more focused scope, as it is applicable to a machine or subsystem in a manufacturing environment.

The seventh and eighth guidelines in the risk analysis journey are referred to as Data Collection and Metrics. This is the stage of analysis where a data collection plan must be developed in order to measure the effects of the risk scenarios on the defined objectives of the system. It is important to formally define the metrics that will be used to evaluate the current state of the system and any progress being made. This is a huge part of a Prognostics and Health Management system. Within the PHM design plan, the Sensing and Feature Analysis component addresses this aspect through the steps of Data Acquisition and Data handling. However, PHM also specifies the use of sensors in order to record data, and furthermore the need to transform the raw data in order to detect degradations in performance. Again, we can see that PHM mirrors the risk analysis guidelines but with a scope that is specifically appropriate for a manufacturing process or system. 
Finally, the ninth and tenth guidelines of risk analysis are Lessons Learned and Guiding Criteria for Decision Making. These final guidelines enter the realm of risk management, where the analysts and stakeholders should evaluate the experiences and results in order to promote an effective decision making process. It is important here to consider the options for what can be done, the tradeoffs between those options, and the potential impacts on any future decisions. PHM also addresses this need for a complete risk evaluation and management plan. The fourth and final PHM design component is titled Response, and it breaks down into a Decision Support aspect and a Presentation aspect. Essentially, the PHM response encompasses a maintenance support plan in order to improve the health and remaining usable life of a manufacturing system's components and subsystems. Similar to risk analysis, PHM also recognizes the need for proper risk communication, which can be facilitated by identifying the key players and stakeholders involved in the system of interest.

\begin{tabular}{|c|c|c|c|}
\hline 1. Emergent Forced Changes & \multirow{4}{*}{$\stackrel{3}{\longleftarrow}$} & \multirow{4}{*}{$\begin{array}{c}\text { PHM Design } \\
\text { Sensing and } \\
\text { Feature } \\
\text { Analysis }\end{array}$} & \multirow{2}{*}{$\begin{array}{l}\text {-Identify High Value Critical } \\
\text { Systems }\end{array}$} \\
\hline 2. "Top Dozen" & & & \\
\hline 3. Variability of the States & & & \multirow{2}{*}{$\begin{array}{l}\text {-Data Acquisition } \\
\text {-Data Handling }\end{array}$} \\
\hline 4. Vulnerability and Resilience & & & \\
\hline 5. Phantom System Models & & \multirow{4}{*}{$\begin{array}{c}\text { Anomaly } \\
\text { Detection \& } \\
\text { Diagnostics }\end{array}$} & \multirow{4}{*}{$\begin{array}{l}\text {-State Detection } \\
\text {-Health Assessment } \\
\text {-Prognostics }\end{array}$} \\
\hline 6. Shared and Unshared States & & & \\
\hline 7. Data Collection & & & \\
\hline 8. Metrics & & & \\
\hline 9. Lessons Learned & \multirow{2}{*}{$\stackrel{\longleftrightarrow}{\longleftrightarrow}$} & \multirow{2}{*}{ Response } & \multirow{2}{*}{$\begin{array}{l}\text {-Decision Support } \\
\text {-Presentation }\end{array}$} \\
\hline 10. Guiding Criteria for Decision Making & & & \\
\hline
\end{tabular}

Figure 3. Visual mapping between Risk Analysis and PHM.

Parts three and four of the Dynamic Roadmap for Risk Analysis contain additional guiding principles and philosophy that harmonize PHM with Risk Analysis. A good way to examine the similarities between these two schools of thought is to break Risk Analysis into two parts: (1) Risk Assessment and (2) Risk Management; and PHM into two parts: (1) Prognostics and (2) Health Management. We can then compare the first parts and the second parts of both fields with one another. 
Part 1 of Risk Analysis has been defined as Risk Assessment, and we are essentially seeking the answers to four major questions (Kaplan \& Garrick, 1981) (Haimes, 2016):

1. What can go wrong?

2. What is the likelihood of that happening?

3. What are the consequences?

4. What is the time frame?

Part 1 of PHM is Prognostics, which means predictive diagnostics. Essentially, this refers to the prediction of the future status, health, or performance of components and systems. In terms of the four questions of risk analysis, prognostics might offer the following questions:

1. Which components or subsystems can become nonoperational?

2. What is the probability of that happening?

3. Which systems will be affected?

4. What is the remaining usable life (RUL) of those components or subsystems?

A quick comparison shows that prognostics and risk assessment are seeking answers to the same types of questions. The only difference is that prognostics is specifically focused on components and subsystems in a manufacturing environment, as well as the negative outcome of an expired RUL.

Part 2 of Risk Analysis can be defined as Risk Management. Again, it can be summed up by seeking to answer three major questions (Kaplan \& Garrick, 1981):

1. What can be done?

2. What are the tradeoffs (costs, benefits, risks)?

3. What are the impacts on future options?

Part 2 of PHM has been defined as Health Management, and again it is essentially the same thing as Risk Management. The central difference between these two definitions is that Health Management in PHM is directly related to decisions in a manufacturing environment, such as operational strategies or maintenance schedules. It is effectively Risk Management but with a 
pre-defined scope. In similar terms as the three risk management questions, health management might ask these questions:

1. What are the possible operational and maintenance strategies?

2. What are the tradeoffs (costs, productivity, risks)?

3. How will the decision impact future operational and maintenance strategies?

By examining some of the fundamental properties, theories, and philosophies of Risk Analysis and Prognostics and Health Management, we can see that these seemingly different schools of thought are actually one and the same. The slight modification is that PHM narrows the scope of Risk Analysis, specifically for a manufacturing process or system. By realizing the similarities between these fields, we can begin to share benefits that each one has to offer. Risk Analysis provides many tools, resources, methodologies, and theories that can advance the fast-growing field of Prognostics and Health Management. This paper offers an altered methodology that combines Hierarchical Holographic Modeling (HHM), Risk Filtering, Ranking, and Management (RFRM), and Fault Tree Analysis (FTA) to help identify the key components and subsystems that would most benefit from PHM within a manufacturing system of systems. 


\section{Part III: Case Studies with Smart Manufacturing Companies}

Case studies with manufacturing companies are a key part of the validation and verification phase of the proposed methodology. They provide real-life evidence that we can truly integrate the field of risk analysis with PHM. Additionally, they allow us to advance from theory and discussion into actual implementation of PHM systems and management strategies. By collaborating with different types of industries, I have refined the established techniques of identifying major precursors to risks. Each case study provides a unique perspective of systemof-systems and unique takeaway lessons.

Due to the competitive nature of the manufacturing industries, their names have been omitted from this paper. The first facility shall henceforth be referred to as Food Company, and the second facility as Tool Company.

\section{Food Company}

\subsection{Food Company - Manufacturing Process Overview}

Food Company is a relatively small-scale manufacturer in the food and food supplement industries. They have developed a complex chemical process to convert inputted raw material into two major products. One of their major systems encompasses the packing, bagging, and transporting of their finished products. Refer to Figure 4 for a detailed system diagram of the entire process with the major components, subsystems, sensors, machines, robots, and humans identified.

Once the product has been processed and fully prepared, it is stored on the floor in a sterilized section of the plant. A small end-loader pushes controlled heaps of the product into a grate in the floor that is outfitted with an automated screw conveyor. This screw moves the product up to a storage tank overhead, which then funnels the product to one of a few possible bagging stations: two smaller stations and one jumbo station for bulk product. After the product enters the funnels, an automated machine fills bags to their correct, preset weight. Bags are administered by human workers, one at each station. The human operators take empty bags, load them onto the bag filler, and then start the filling process. Finally they remove the full bags and shift the bags over to a conveyor where they are sealed, flattened, and sent down the line. 
At this point the bags are in queue for a robotic palletizer. The palletizer receives sealed and inspected bags of product and stacks them onto wooden pallets in regular, repeating patterns that can be selected and adjusted by the operator. A forklift is used to remove the finished pallet where it is wrapped in shrink wrap and placed in a holding area for distribution. A central programmable logic controller with a touch screen interface coordinates the overall unit automation that was supplemented by at least six human workers: one end-loader driver, two baggers, one inspector, and two to shrink wrap finished pallets and insert empty pallets to the palletizer cage. 

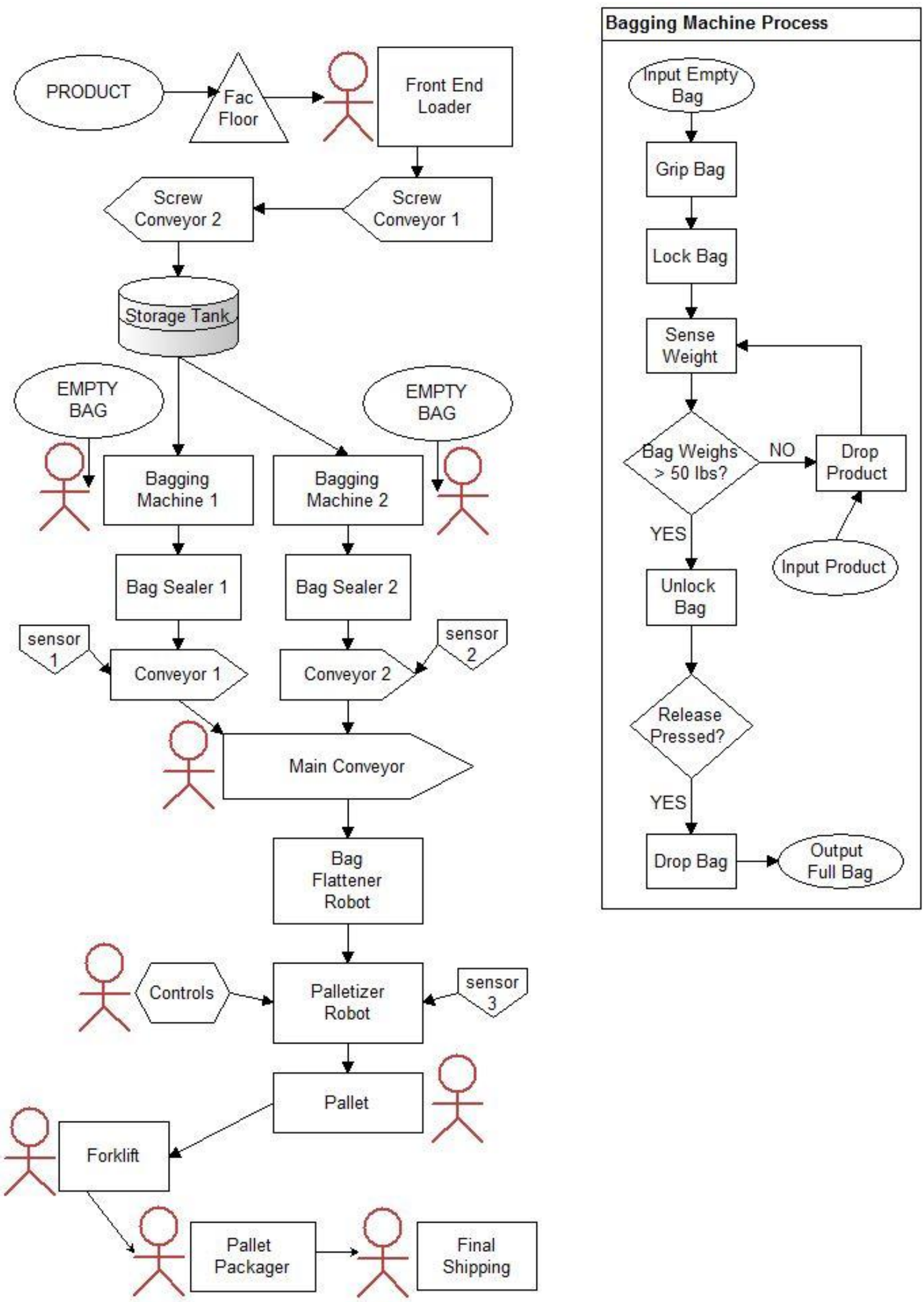

Figure 4. System diagram of Food Company's product packing and bagging process. 


\subsection{Food Company - Objectives and Known Issues}

The main objectives for Food Company are to maximize production of their food product, and to minimize the risk of a system failure (production shutdown or delay). To help achieve these objectives, the plant managers wish to implement a PHM system into their product packing and bagging process. However, they currently have limited monetary resources allocated towards this project. Therefore, Food Company has asked for a risk analysis consultation regarding which of their components/machines/subsystems would most benefit from a PHM system. This necessitates a complete understanding of their current industrial process.

Plant engineers and managers have identified the following known health management issues:

- The funnel openings can become clogged with finished food product if not regularly cleaned out.

- Sensors fail with regularity. Common causes of failure include occlusion of optical components by dirt and misalignment through collision with bags of product.

- The maneuvering of heavy bags by human workers is a potential source of slower productivity for the facility.

- Product sitting out in the open on the warehouse floor can become contaminated.

- Adjusting and reprogramming the palletizer is difficult and generally outside the scope of the work done in house. The robot engineer must be on call and able to reprogram the machine in-person.

\section{Tool Company}

\subsection{Tool Company - Manufacturing Process Overview}

Tool Company is a relatively large-scale manufacturer of tools and tooling systems designed for advanced industrial metal cutting. One of their machining groups is divided into two major production lines: Standard and Specials. The Standard line focuses on the manufacturing of a limited number of tools and products that are featured in their catalog. The Specials line on the other hand is a flexible business unit that handles unique, custom order designs from clients that are not featured in their catalog. Both production lines are further divided into three product lines or "flow groups". The Drilling group produces drill bits and tools used for tapping; the Milling 
Cutter group produces cutting tools for milling process; and the Boring Bar group produces tools for lathes.

The Tool Company Standard line is a prime candidate for extensive Risk Modeling and for deploying a Prognostic and Health Management (PHM) system. The Standard line encompasses a limited number of processes that repeat periodically and have been optimized to some extent by Tool Company operators and engineers. However, a PHM system could provide additional information regarding the present and future status, health, or performance of some of the major components, machines, and robots involved in the processes. This would help to improve production output, maintenance scheduling, and planning for downtime or ordering replacement parts. Additionally since the Standard line repeats processes, data from a PHM system can be extracted to make weekly, monthly, or yearly predictions.

The production phase of the Tool Company Standard line is mirrored across the Drilling, Milling Cutter, and Boring Bar flow groups. All orders follow the following general processes: cutoff saw, heat treatment, lathe operation, grinder, 5-axis processing, black oxide coating, assembly, labeling \& packaging, and final inspection, as can be seen in Figure 5. It was noted that problems were more likely to occur at the cutoff saw, lathe operation, grinder, and 5-axis processing stations due to variance in operators, cutting tools, machines, and raw material. 


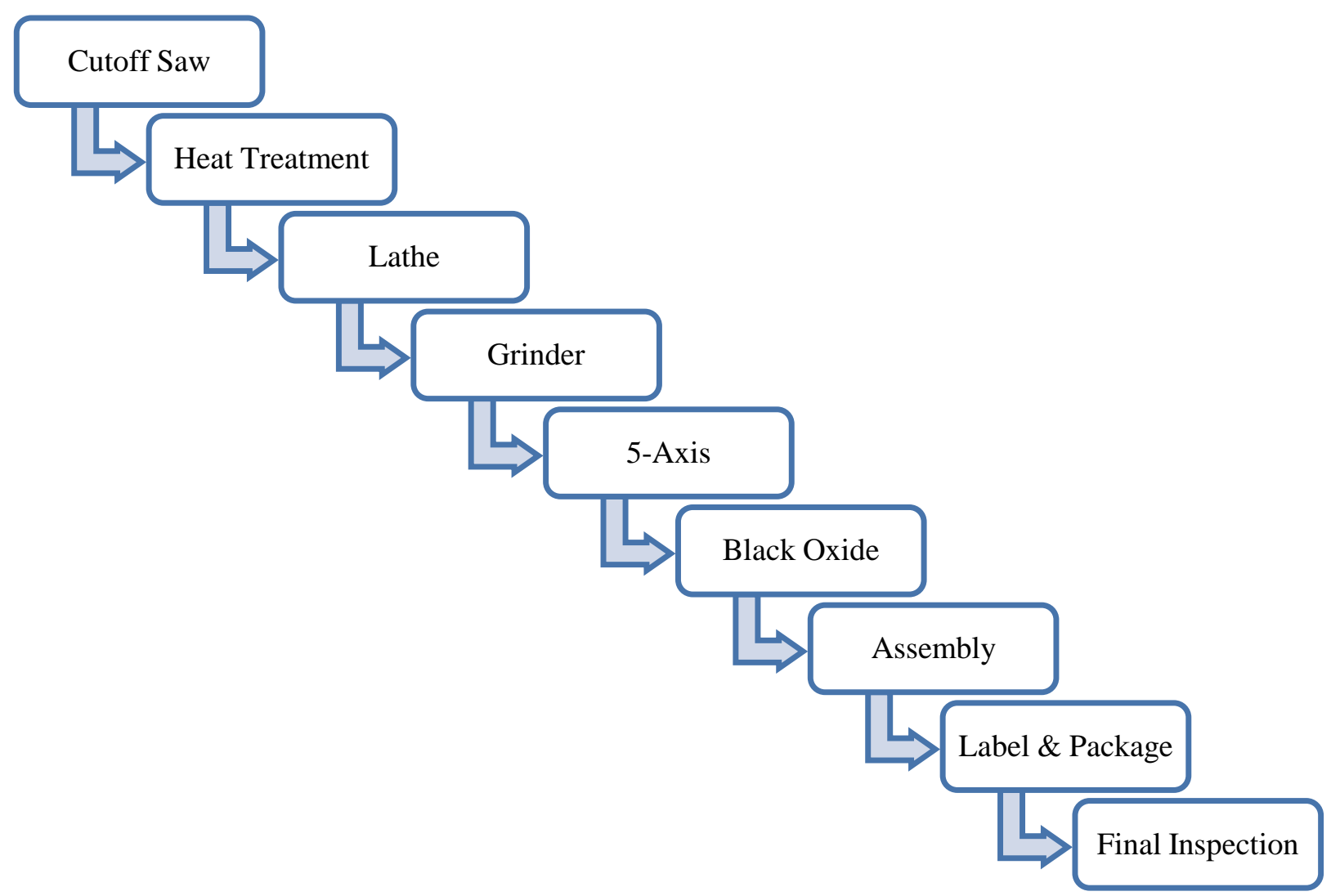

Figure 5. Sandvik Standards line production processes.

\subsection{Tool Company - Objectives and Known Issues}

Tool Company currently follows a scheduled maintenance plan with a reactive maintenance policy where they attend to problems as they arrive. However, they are very interested in implementing a predictive maintenance policy, especially in their Standard line where jobs are repeated and the wear on their machines can be observed and recorded. PHM systems provide this exact type of capability with sensing, simulation, and automation. Unfortunately, there are many different components within the Standard line that might be viable areas to implement such a system. Therefore, Tool Company is interested to determine a set of top targets within their manufacturing process that would most benefit from PHM. We have been asked to perform risk analysis, modeling, and assessment for Tool Company with the primary objective being to maximize production, and with the end goal of providing scope for a PHM system. 
Plant engineers and managers have identified the following known issues with their manufacturing process:

- Overall Equipment Effectiveness (OEE) - a metric used to evaluate the efficiency of a machine by combining product availability, quality, and throughput - was lower than desired.

- Some of the production machines (e.g., 5-axis machine, lathe, and grinder) have components that wear out and are subject to more downtime.

- Software for tracking maintenance requests is still missing information for understanding the root cause and full effect of an incident.

- Operators may have different priorities and incentives than management.

- Operators and engineers may design programs to operate at different speeds and settings for similar jobs.

\section{Major Lessons Learned}

The case studies with the Food Company and the Tool Company helped to solidify the harmonization between the fields of Risk Analysis and Prognostics and Health Management. I successfully developed a modified risk methodology for identifying key components in a manufacturing environment that would most benefit from PHM system capabilities. By conducting multiple studies, we were able to demonstrate that the methodology is compatible with manufacturing companies of different industries and of varying scales of production.

After presenting the studies back to the original companies, their managers and decision makers agreed that some of the findings were unexpected, and that they would begin implementing solutions towards managing the top identified risk scenarios. The top risk scenario with the Food Company was the failure of their Palletizing Robot, and after a fault tree analysis we identified the major components that needed to be monitored. Similarly, the top risk scenario with the Tool Company was the failure of their 5-Axis Machine. The fault tree analysis also pointed out the minimal cut set of components that would most benefit from PHM capabilities. 


\section{Part IV: Methodological Framework: Systems-Based Risk Analysis}

\section{Hierarchical Holographic Modeling (HHM)}

\subsection{Definition of HHM}

Hierarchical Holographic Modeling (HHM) is a holistic philosophy and methodology in the field of Risk Analysis that considers all possible sources of risk (e.g., hardware, software, organizational, human, cultural, and external). It aims to capture and represent the inherent diverse characteristics and attributes of a system, including its multiple aspects, perspectives, views, dimensions, and hierarchies (Haimes 1981, 2008, 2012). HHM models are developed within an organized team of system experts containing varied experiences and knowledge bases. The actual models are comprised of head topics (representing different categories for sources of risk to propagate) and then sub topics which contain the details and components of these potential risks. Each head topic and sub topic has the potential to be expanded into an HHM model in and of itself, leading to even more levels and insight into the system's intrinsic hierarchy.

The hierarchical aspect of HHM models refers to the multiple levels that are present within a large-scale system-of-systems. In the field of smart manufacturing, these levels can usually be represented as follows:

2. System (e.g., factory)

a. Subsystem (e.g., work station)

i. Machine (e.g., lathe)

1. Component (e.g., spindle)

a. Primitive (e.g., cutting insert)

The holographic aspect of HHM models refers to the desire to have a multi-view image of a system. This can be accomplished by creating multiple HHM models with different categories for the head topics. For example, the risks to a system can be divided by geographic, economic, health, technical, political, social, or organizational factors. In order to properly capture these many views, the modeling team must represent a broad and extensive array of experience and knowledge. 


\subsection{Benefit of HHM}

The process of developing HHM models is an essential first-step in any major risk analysis and modeling effort. HHM models help to ensure that the system of interest has been represented in its entirety, and that all major sources of risk have at least been considered. Additionally, an HHM model can be used to determine the reliability and maintainability of a system or an infrastructure featuring a large number of subsystems and components. It accomplishes this by revealing the dependencies and overlapping between subtopics, which can be analyzed to show the multiple paths to a specific failure and the cascading effects from a failure. From a mathematical standpoint, reliability refers to the "probability that a system is operational in a given time period." Maintainability on the other hand is defined as the "probability that a failed system can be restored to an operational state within a specified period of time" (Haimes, 2009). Both of these metrics are essential to holistic risk management and to Prognostics and Health Management.

The Hierarchical Holographic Modeling process is especially useful because it considers risks at both the macroscopic (management) and microscopic (component) levels. Most organizational and technology-based manufacturing systems are hierarchical in nature (Alvandi, Bienert, Li, \& Kara, 2015) (He, Zhang, \& Li, 2014), and the deployments of HHM have effectively addressed the risks at these multiple levels (Haimes, Kaplan, \& Lambert, 2002). During the decomposition process of HHM, two major types of risks will ultimately come to light: those resulting from 1) exogenous events such as new legislation or natural disasters, and 2) endogenous events such as hardware, software, organizational, and human failures (Haimes, 2009). While knowledge of both types of events is crucial to understanding the entire system, a PHM strategy will rely more heavily on the potential endogenous events which are more addressable.

The HHM methodology produces a visual decomposition of a system into its many subsystems and components. This breakdown is essential to revealing the complexity and internal hierarchical nature of large-scale systems (He et al., 2014). Decomposition also allows for tradeoff analyses and studies to be performed at the component, subsystem, or total system level. Applying the HHM methodology requires an organized team of experts with varied experience and knowledge bases to develop a holographic view of a system with its multiple levels and 
hierarchies. Although it is possible for individual experts to create different decompositions, the aggregate will yield the same optimal solution. Each expert will provide their own perspective to enforce the desired multi-view image of the system and reveal unique vulnerabilities (Kaplan, Haimes, \& Garrick, 2001). Two major types of risks and uncertainties will ultimately come to light: those resulting from 1) exogenous events such as new legislation or natural disasters, and 2) endogenous events such as hardware, software, organizational, and human failures (Haimes, 2009). While knowledge of both types of events is crucial to understanding the entire system, a PHM system will focus more heavily on potential endogenous events which can take the form of critical EFCs.

\subsection{Flipping the HHM}

The act of "flipping" the HHM is essential to a holistic risk modeling process. It allows the analysts and stakeholders to examine a system-of-systems from a brand new perspective. Flipping the HHM simply means examining an existing Hierarchical Holographic Model with a new type of decomposition. Example decompositions for these models include (but are not limited to) technical, social, environmental, geographic, organizational, product, and temporal.

An HHM model should only be "flipped" after the experts and stakeholders agree that the current model represents all possible risk scenarios, or at least all of the important ones. The act of creating a new model with a new decomposition gives the potential for additional risk scenarios to be realized. When creating a system model using a new perspective, the experts may include additional subsystems or components that did not fit nicely in the original HHM. This is especially important when trying to consider everything that could go wrong during the planning phase of a new operation. This idea is further illustrated within the Sandvik and Omega Protein case studies. 


\subsection{Risk Survey with Experts}

One of the major difficulties with the development process of a Hierarchical Holographic Model is that it requires joint collaboration with multiple experts from a diverse set of backgrounds. In order to facilitate this process, a risk analysis survey was developed titled Risk in Manufacturing Processes. The survey is the outcome of a collaborative effort between our UVA research team, NIST, industry experts, the UVA Center for Survey Research, and the UVA Institutional Review Board for Social and Behavioral Sciences. The purpose of the survey is to extract all relevant information from experts regarding system risks to a manufacturing process but on an individual basis and in a timely manner (less than half an hour). The survey also ensures that everyone is exposed to the same questions to guarantee consistency and allow everyone to have an equal impact. It also allows for manufacturing companies to conduct the survey with their employees on their own without the direct need for a risk analysis facilitator. The additional value of the survey shall be measured and validated through case studies with the manufacturing companies "Food Company" and "Tool Company". See Appendix A. Risk in Manufacturing Processes Survey for a complete copy of the survey.

\subsection{Food Company - HHM}

The initial HHM model for Food Company was developed with the perspective/decomposition of the different physical components within the finished product bagging system. The head topics for the model were (1) Processing Machines, (2) Transportation Machines, (3) Product Components, (4) Humans, and (5) Environment. Underneath these major topics, subtopics and eventually potential risk scenarios were identified. The HHM model for the physical components of Food Company can be seen in Figure 6. 


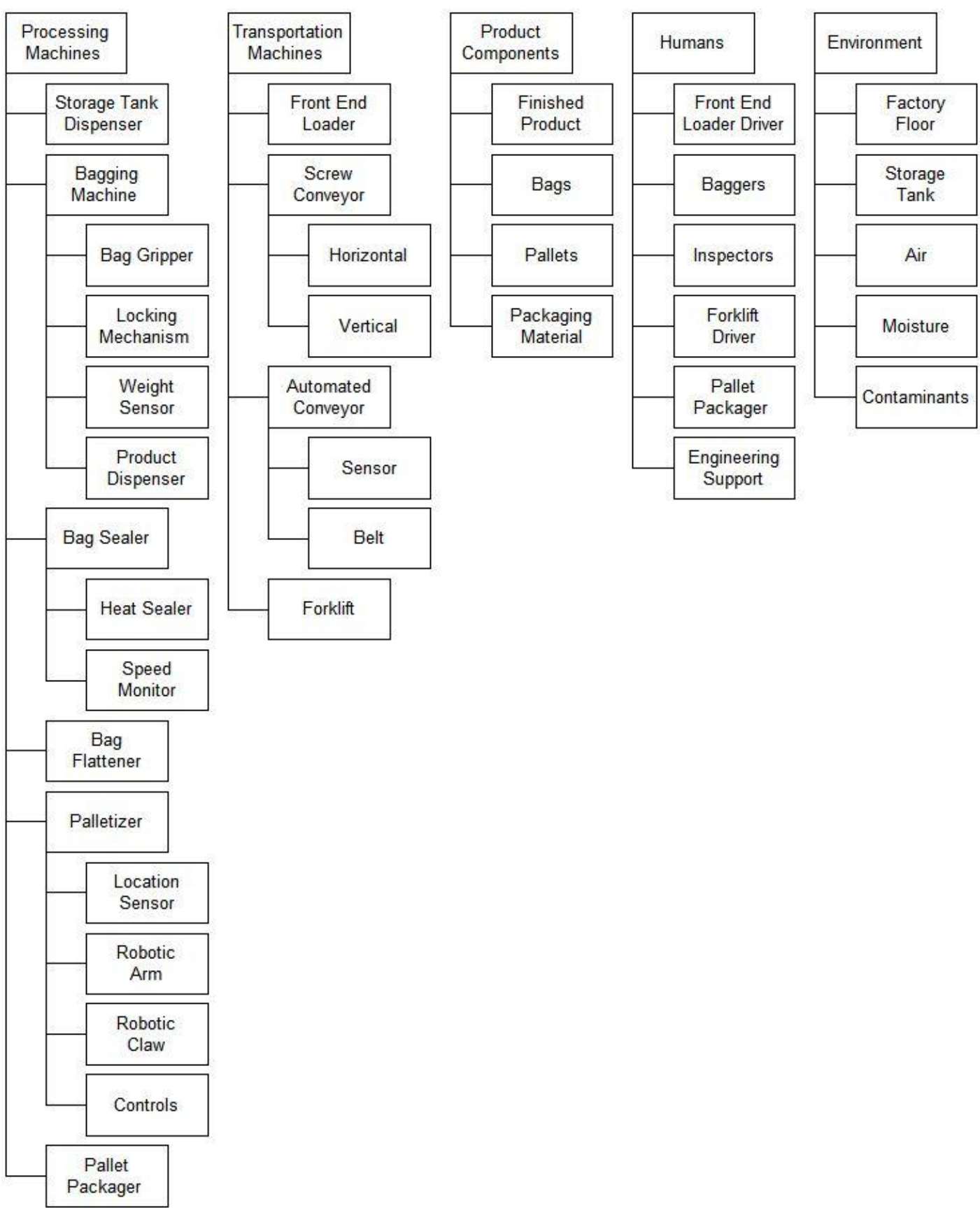

Figure 6. HHM for Food Company - Physical Components decomposition.

A similar HHM model was also developed from multiple Food Company experts covering a new perspective/decomposition: the different processes involved in the fish meal bagging system. This second model was created with the idea of "flipping" the original model and checking for new sources of risk that may have originally been missed. In fact, just the practice of creating multiple HHM models helps to provide a holographic view of the entire system and ensure that 
the major sources of risk are properly captured. It provides a more realistic and complete overall model by recognizing the limitations of modeling a complex system with just a single structure. The head topics for the processes model were (1) Storing Product, (2) Transporting Product, (3) Bagging Product, (4) Sealing Bags, (5) Transporting Bags, (6) Flattening Bags, (7) Palletizing Bags, and (8) Final Preparation for Delivery. The complete HHM model for the processes at Food Company can be seen in Figure 7.

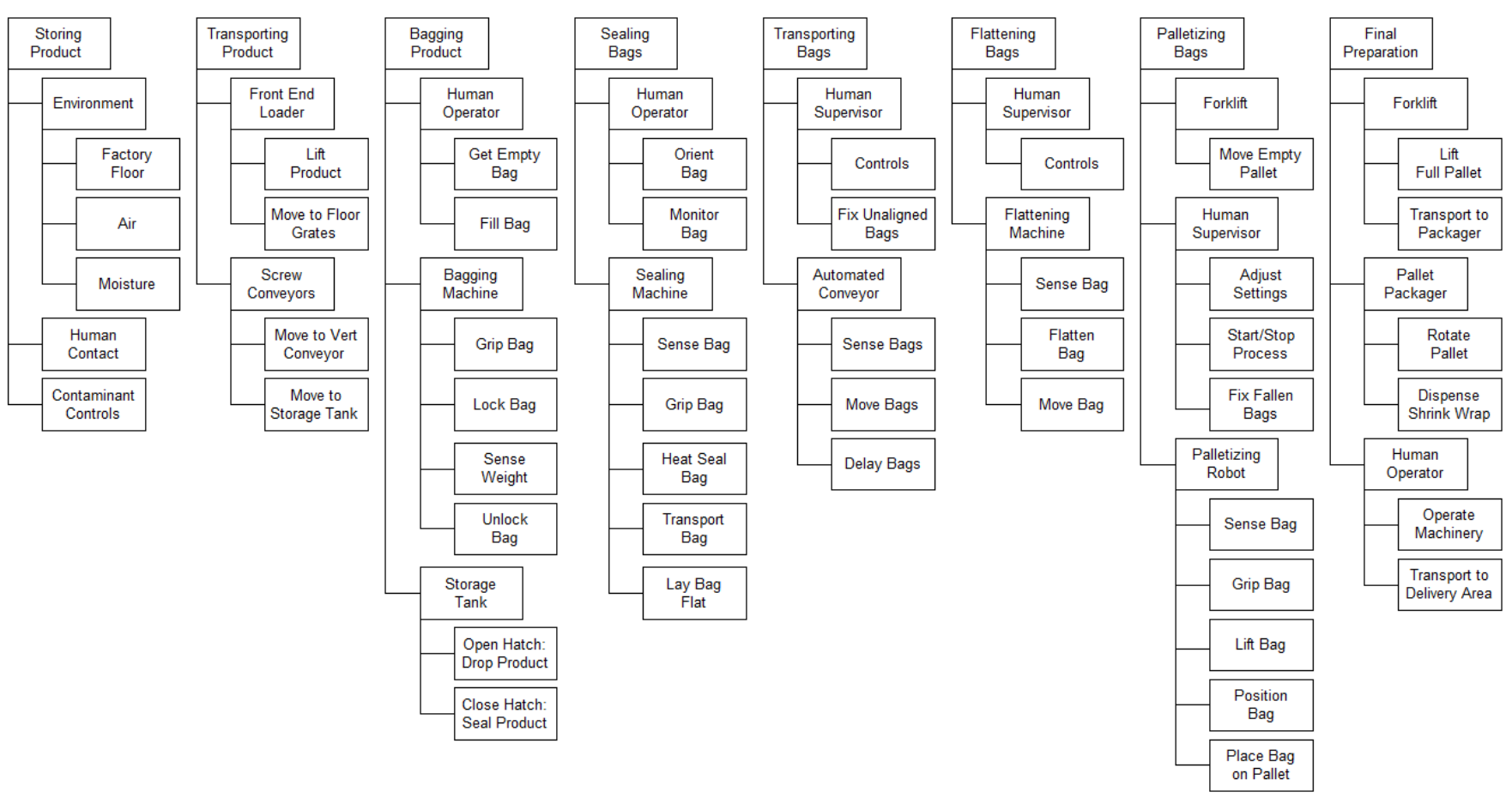

Figure 7. HHM for Food Company - Processes decomposition.

Additional HHM perspectives can always be explored to further improve the overall system model, such as organizational, technological, or even social. For this particular case study, the processes perspective was used to develop risk scenarios for the fish meal packing and bagging system at Food Company. 


\subsection{Tool Company - HHM}

The Tool Company Standard line integrates multiple business groups and subsystems in order to function properly. Therefore, following the risk analysis methodology, we start with a top-level Hierarchical Holographic Model. As with any HHM, the analysts have multiple options for the type of decomposition to select for the model. For the study with Tool Company the following decompositions were suggested: Operational, Resource, Societal, Technical, Customer, Political, Environmental, Managerial, Financial, Infrastructure, External, Product, Geographical, Cultural, and Temporal. In this case, the Operational perspective was selected, and the head topics represented the various departments at Tool Company. These major head topics identified were as follows: (1) Production; (2) Engineering; (3) Maintenance; (4) Logistics; (5) Management; (6) Environment, Health, and Safety (EHS); and (7) Quality. This is a very logical decomposition for the hierarchical breakdown of a manufacturing company, since many follow similar organizational structures. The HHM model is shown in Figure 8, and represents the interconnected and interdependent operational subsystems of Tool Company.

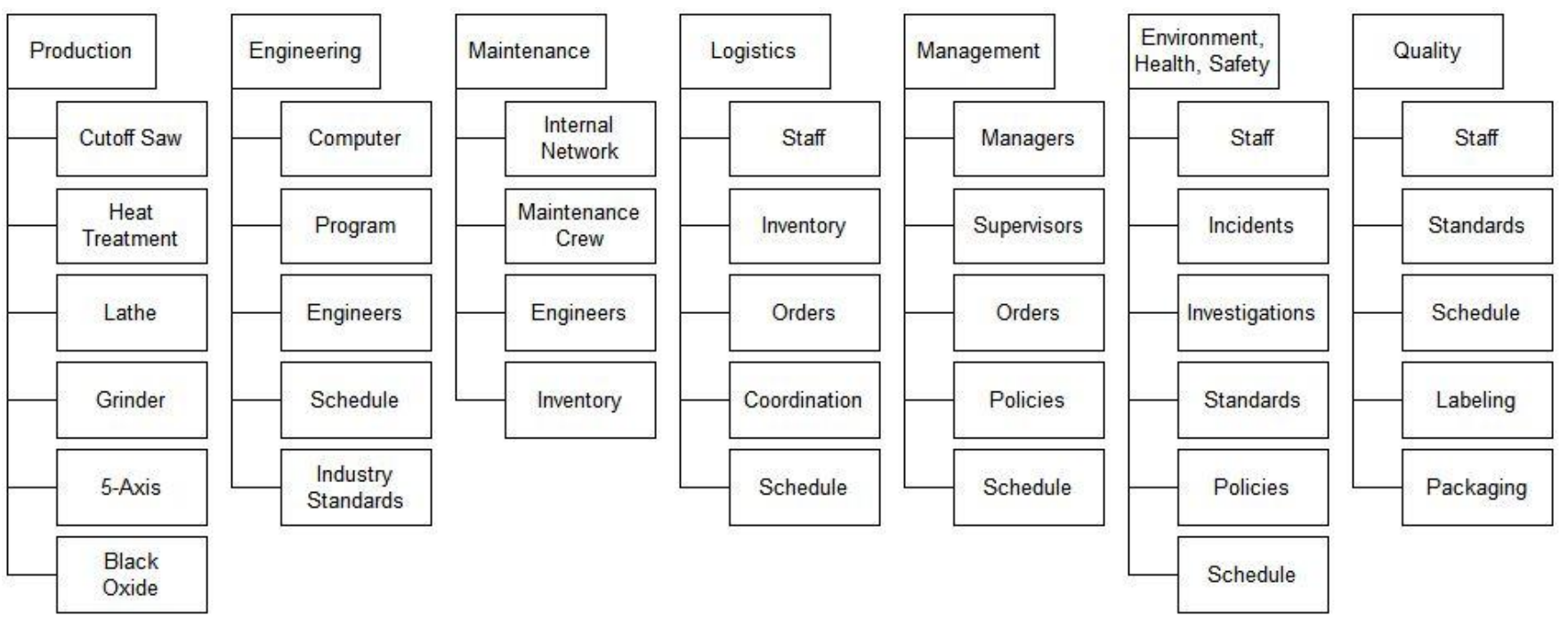

Figure 8. HHM for Tool Company - Operational decomposition.

However, since in this particular case study we have defined our major objective as being to maximize production, we decided to create a more detailed Hierarchical Holographic Model underneath the Production head topic. This is essentially a subsystem HHM within the larger system HHM. Our new head topics for the model are therefore the subtopics underneath 
"Production" from the top level Operational decomposition model: (1) Cutoff Saw, (2) Heat Treatment, (3) Lathe, (4) Grinder, (5) 5-Axis Machine, and (6) Black Oxide Treatment. Clearly since these new head topics are all machines in the production process, this new HHM model has a Machine perspective. The detailed HHM model for Tool Company using the Production decomposition with a Machine perspective can be seen in Figure 9.

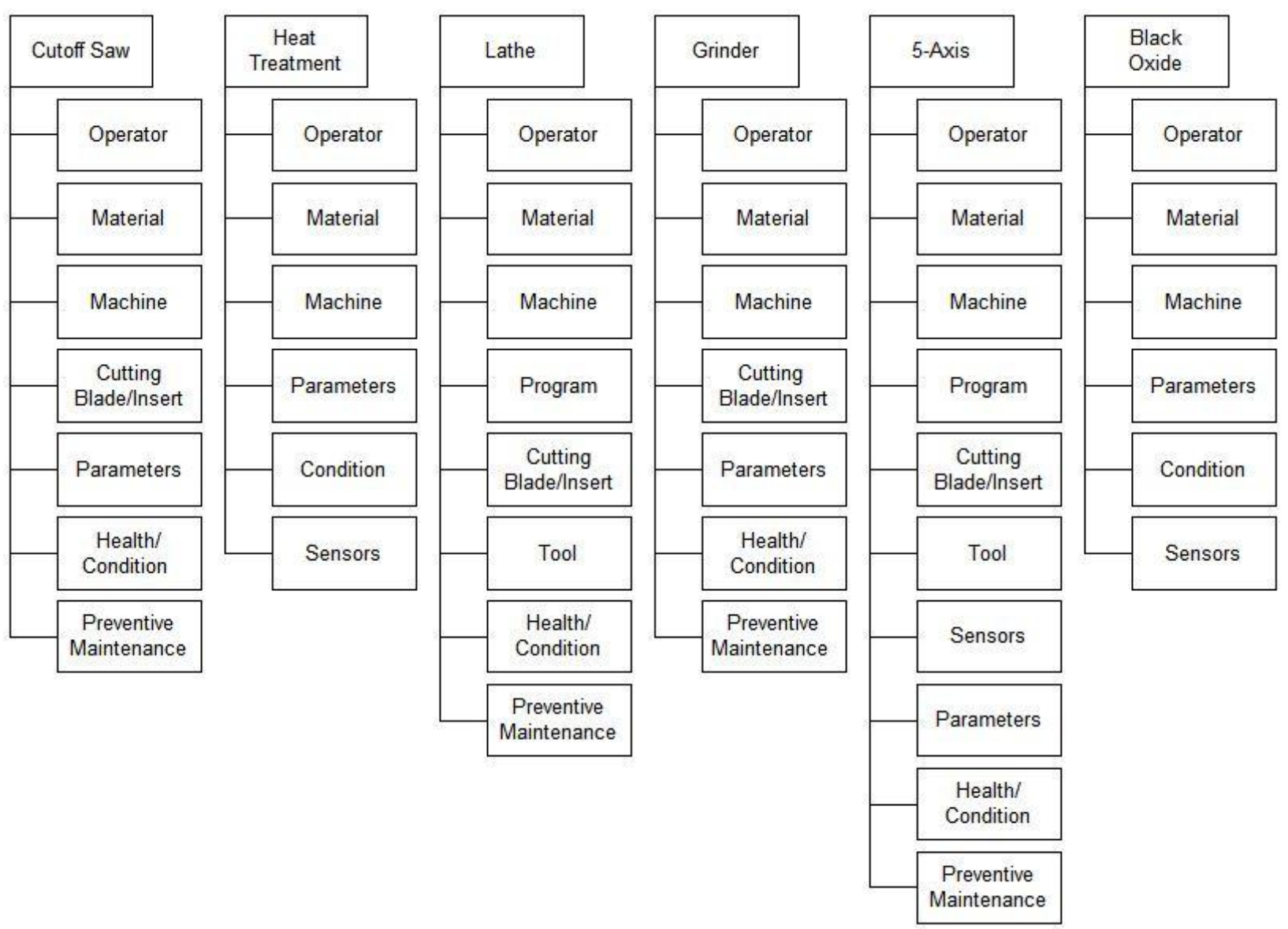

Figure 9. HHM for Tool Company - Production decomposition with Machine perspective.

The final step in the HHM modeling process is to "flip" the HHM model using a new perspective, such as by type of Resource. This is an extremely useful tool during the scenario identification phase to ensure that all relevant risks have been realized. The flipped Hierarchical Holographic Model for Tool Company using the Production decomposition and with a Resource perspective can be found in Figure 10. 


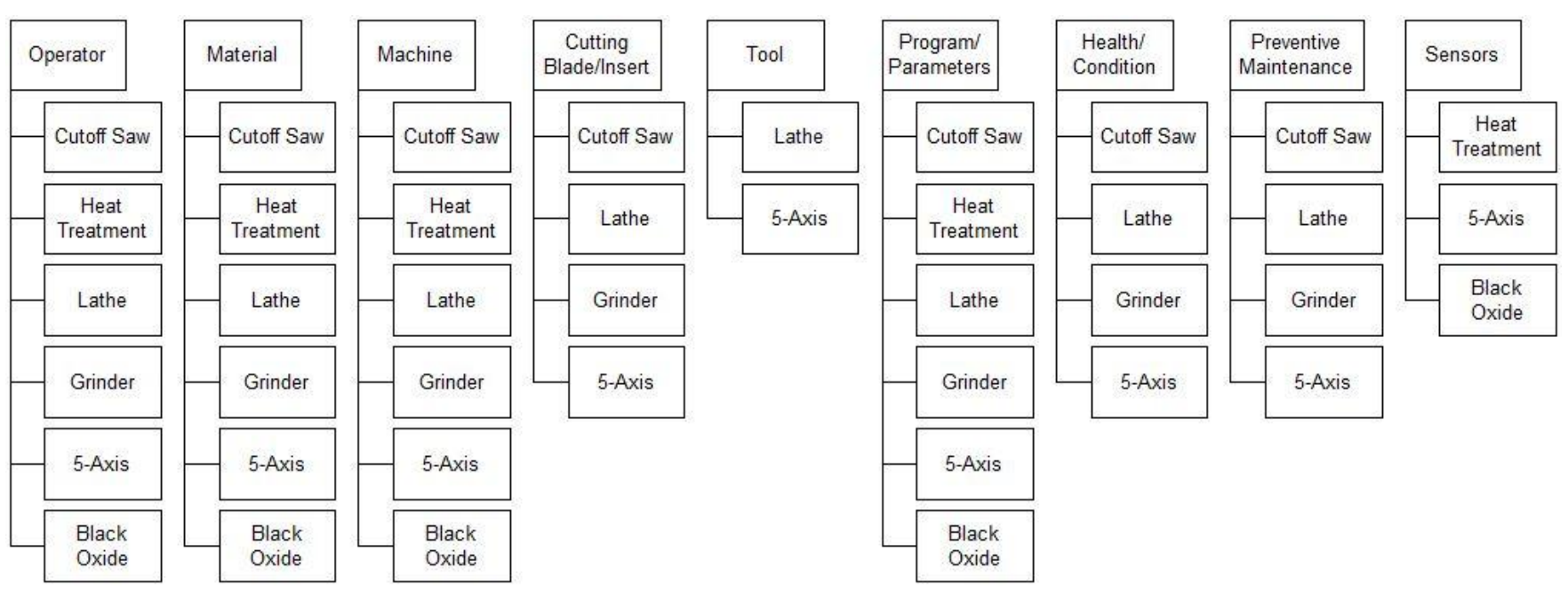

Figure 10. HHM for Tool Company - Production decomposition with Resource perspective.

It should be noted that in this case the flipped HHM model (refer to Figure 10) is identical to the original one (refer to Figure 9) regarding content. The only difference is that the head topics and subtopics have been swapped. This helps to provide a unique perspective to the risk analysts to understand how risks may propagate up through the system. Additional HHM perspectives can always be explored to further improve the overall system model. However, once the analysts and experts are satisfied with their model, they can begin developing risk scenarios for the system and proceed to the Risk Filtering, Ranking, and Management methodology. 


\section{Risk Filtering, Ranking, and Management (RFRM)}

\subsection{Definition and Benefit of RFRM}

Risk Filtering, Ranking, and Management (RFRM) is a risk analysis methodology encompassing the identification, prioritization, assessment, and management of potential risk scenarios to a large-scale system. Stakeholders and decision-makers must consider the likelihoods and consequences of each risk to produce acceptable mitigation options. The Risk Filtering, Ranking, and Management (RFRM) methodology offers eight major phases to guide total risk management in an HHM system (Haimes, 2002). The eight phases are: (1) Scenario Identification, (2) Scenario Filtering, (3) Bi-criteria filtering, (4) Quantitative Ranking, (5) Multicriteria Evaluation, (6) Risk Management, (7) Safeguarding Against Missing Critical Items, and (8) Operational Feedback. Details on these eight phases can be found in (Haimes, 2002). For the case studies with the manufacturing companies, we focused on phases 1 through 5 . Phases 6 through 8 were incorporated later through risk mitigation strategies and management plans.

The guiding force behind RFRM is the identification of all major risk scenarios that could negatively impact the system. It is important for analysts to consider a variety of risks, such as those related to hardware, software, organizational failure, human error, budget, schedule slip, and various performance criteria (Haimes, 2002). However, a successful identification phase relies on the previous development of an accurate Hierarchical Holographic Model (HHM), complete with head topics and relevant subtopics (Haimes, 2009). While identifying major risk scenarios, it is not uncommon for analysts to find themselves with hundreds of unique scenarios. Since it is often impractical and infeasible to analyze hundreds of sources of risk, the scenarios must be prioritized and thus filtered based on their likelihood (probability) and impact (severity) to the overall system. These qualities can be determined base on professional experience, expert knowledge, and statistical data (Haimes, 2002). 
The risk assessment portion of RFRM can be approached by seeking the answers to four major questions (Kaplan \& Garrick, 1981) (Haimes, 2016):

1) What can go wrong?

2) What is the likelihood of that happening?

3) What are the consequences?

4) What is the time frame?

The risk management portion on the other hand encompasses three complementary questions (Kaplan \& Garrick, 1981) (Haimes, 2016):

1) What can be done and what are the available options?

2) What are the associated trade-offs in terms of costs, benefits, and risks?

3) What are the impacts of current decisions on future options?

\subsection{RFRM Phases 1 \& 2: Scenario Identification / Scenario Filtering}

Following the Risk Filtering, Ranking, and Management methodology, the first phase is to identify all possible risk scenarios to the system of interest. This at first appears to be a very difficult process, but it is simplified by using the previously developed Hierarchical Holographic Models. From the models, each head topic represents a category of risk scenarios for the experts to identify and formally define. Then the subtopics provide guidance for the specific details about the different risk scenarios for each identified category. Following this process helps to ensure that no major risks are missed or overlooked by the experts.

The second phase of RFRM is to perform Scenario Filtering based on scope, temporal domain, and level of decision making. This is a crude filtering step in order to reduce the initial number of risk scenarios under consideration (potentially from over 100 down to 10-20). This step is expedited using the Risk in Manufacturing Processes Survey discussed in the HHM section (refer to Appendix A. Risk in Manufacturing Processes Survey). From the survey, major risk scenarios that should be considered are described and often repeated by multiple experts. 


\section{2a Food Company - Scenario Identification / Scenario Filtering}

For the case study with Food Company, risk scenarios were first identified using the HHM model with the processes decomposition (refer to Figure 7). Each head topic was transformed into a risk category, and subtopics were transformed into the specific risk scenarios. The complete list of risk scenarios (about 75) can be found in Appendix B. Food Company - Full List of Risk Scenarios. However, after a basic filtering by the experts/analysts based on scope, temporal domain, and level of decision making, the list has been reduced to the twelve risks shown in Table 1. This reduced list is often referred to as the "Top Dozen". It should be noted that the Risk IDs for each scenario correspond to the location of the risk on the original HHM model.

Table 1. Top risk scenarios of interest for Food Company.

\begin{tabular}{|l|l|l|}
\hline Risk ID & Subsystem/Process & Risk Description \\
\hline 1.a & Storing Product & Environment Failure \\
\hline 2.a & Transporting Product & Front End Loader Failure \\
\hline 2.b & Transporting Product & Screw Conveyors Failure \\
\hline 3.a & Multiple Processes & Human Operator Failure \\
\hline 3.b & Bagging Product & Bagging Machine Failure \\
\hline 3.c & Bagging Product & Storage Tank Failure \\
\hline 4.b & Sealing Bags & Sealing Machine Failure \\
\hline 5.b & Transporting Bags & Automated Conveyor Failure \\
\hline 6.b & Flattening Bags & Flattening Machine Failure \\
\hline 7.a & Palletizing Bags & Forklift Failure \\
\hline 7.c & Palletizing Bags & Palletizing Robot Failure \\
\hline 8.b & Final Preparation & Pallet Packager Failure \\
\hline
\end{tabular}

\section{2b Tool Company - Scenario Identification / Scenario Filtering}

For the case study with Tool Company, risk scenarios were initially identified using a combination of the top level HHM model featuring the operational decomposition (refer to Figure 8), along with the detailed HHM model for the production decomposition with the machine perspective (refer to Figure 9). Head topics, sub topics, and sub-sub topics were transformed into specific risk scenarios for the manufacturing process. The complete list of risk scenarios (over 100) can be found in Appendix C. Tool Company - Full List of Risk Scenarios. Again, after basic filtering by experts/analysts based on scope, temporal domain, and 
level of decision making, the list has been reduced to the twelve risks shown in Table 2. The Risk IDs for each scenario correspond to the location of the risk on the original HHM model.

Table 2. Top risk scenarios of interest for Tool Company.

\begin{tabular}{|l|l|l|}
\hline Risk ID & Subsystem & Risk Description \\
\hline $1 . \mathrm{a}$ & Production & Cutoff Saw Failure \\
\hline $1 . \mathrm{b}$ & Production & Heat Treatment Failure \\
\hline $1 . \mathrm{c}$ & Production & Lathe Failure \\
\hline $1 . \mathrm{d}$ & Production & Grinder Failure \\
\hline $1 . \mathrm{e}$ & Production & 5-Axis Failure \\
\hline $1 . \mathrm{f}$ & Production & Black Oxide Failure \\
\hline $1 . \mathrm{g}$ & Production & Wrong Tool/Program \\
\hline 2.b & Engineering & Program Failure \\
\hline 3.a & Maintenance & Internal Network Failure \\
\hline 4.b & Logistics & Missing Tools/Materials \\
\hline $5 . \mathrm{e}$ & Management & Missing Human Support \\
\hline $6 . \mathrm{b}$ & EHS & Accident/Incident \\
\hline
\end{tabular}

\subsection{RFRM Phases 3 \& 4: Bi-Criteria Filtering / Quantitative Ranking}

Phases 3 of the RFRM methodology involves filtering and ranking the risk scenarios based on their likelihood (probability) and impact (severity). This can be accomplished by placing the scenarios on a qualitative severity-scale matrix, and then filtering out the risks that do not meet the predetermined risk threshold. The matrix used in this process has been adapted from the Military Standard 882, U.S. Department of Defense (Roland \& Moriarty, 1990). Table 3 is an example of the matrix used in Phase 3. The different impact levels are established by the decision makers and risk analysts for the system or facility of interest. 
Table 3. Example risk matrix for RFRM Phase 3.

\begin{tabular}{|c|c|c|c|c|c|}
\hline \multirow{2}{*}{ Impact } & \multicolumn{5}{|c|}{ Likelihood/Probability } \\
\cline { 2 - 5 } & Unlikely & Seldom & Occasional & Likely & Frequent \\
\hline Production & & & & & \\
Shutdown & & & & & \\
\hline Loss of & & & & & \\
Product & & & & & \\
\hline Reduced & & & & & \\
Speed & & & & & \\
\hline Minor & & & & & \\
Degradation & & & & & \\
\hline Minor or & & & & \\
No Effect & & & & & \\
\hline
\end{tabular}

\begin{tabular}{|c|c|c|c|}
\hline Low & Moderate & High & Extremely \\
Risk & Risk & Risk & High Risk \\
\hline
\end{tabular}

Risks that are placed in the upper right hand corner of the matrix should be considered with higher priority than those in the lower left. It is common procedure to only advance the scenarios placed in the "High Risk" and "Extremely High Risk" categories through the rest of the filtering and ranking methodology. Phase 4 is the exact same procedure as Phase 3, but with probability ranges assigned to the qualitative likelihood terms. For this reason, these steps are often combined and performed simultaneously. The probability ranges are subject to change by the decision makers and analysts, but typical values are as follows:

- Unlikely: $0-1 \%$

- Seldom: $1-5 \%$

- Occasional: 5-10\%

- Likely: $10-50 \%$

- Frequent: $50-100 \%$ 


\section{3a Food Company - Bi-Criteria Filtering / Quantitative Ranking}

Back to the case study with Food Company, we follow Phases 3 and 4 of the RFRM methodology by placing the top identified risk scenarios in a severity-scale matrix, with the likelihoods defined by ranges of probabilities. The results of the matrix can be seen in Table 4 .

Table 4. Risk matrix for Food Company.

\begin{tabular}{|c|c|c|c|c|c|}
\hline \multirow{2}{*}{ Impact } & \multicolumn{5}{|c|}{ Likelihood/Probability } \\
\cline { 2 - 6 } & $0-1 \%$ & $1-5 \%$ & $5-10 \%$ & $10-50 \%$ & $50-100 \%$ \\
\hline $\begin{array}{c}\text { Production } \\
\text { Shutdown }\end{array}$ & $\mathbf{7 . c}$ & & & \\
\hline $\begin{array}{c}\text { Loss of } \\
\text { Product }\end{array}$ & $\mathbf{3 . c}$ & $\mathbf{2 . b}$ & $\mathbf{3 . a , 3 . b}$ & & \\
\hline $\begin{array}{c}\text { Reduced } \\
\text { Speed }\end{array}$ & $\mathbf{2 . a , 7 . a}$ & $\mathbf{5 . b , 8 . b}$ & & $\mathbf{6 . b}$ & \\
\hline $\begin{array}{c}\text { Minor } \\
\text { Degradation }\end{array}$ & $\mathbf{1 . a , 4 . b}$ & & & & \\
\hline $\begin{array}{c}\text { Minor or } \\
\text { No Effect }\end{array}$ & & & & & \\
\hline
\end{tabular}

\begin{tabular}{|l|c|c|c|}
\hline Low & Moderate & High & Extremely \\
Risk & Risk & Risk & High Risk \\
\hline
\end{tabular}

Taking only the risks that were placed in the "High Risk" and "Extremely High Risk" categories, our new list of scenarios can be seen in Table 5.

Table 5. Reduced list of risk scenarios for Food Company.

\begin{tabular}{|l|l|l|}
\hline Risk ID & Subsystem/Process & Risk Description \\
\hline 3.a & Multiple Processes & Human Operator Failure \\
\hline 3.b & Bagging Product & Bagging Machine Failure \\
\hline 6.b & Flattening Bags & Flattening Machine Failure \\
\hline 7.c & Palletizing Bags & Palletizing Robot Failure \\
\hline
\end{tabular}

\section{3b Tool Company - Bi-Criteria Filtering / Quantitative Ranking}

For the case study with Tool Company, we also follow Phases 3 and 4 of the RFRM methodology by placing the top identified risk scenarios in a severity-scale matrix, with the likelihoods defined by ranges of probabilities. The results of the matrix can be seen in Table 6 . 
Table 6. Risk matrix for Tool Company.

\begin{tabular}{|c|c|c|c|c|c|}
\hline \multirow{2}{*}{ Impact } & \multicolumn{5}{|c|}{ Likelihood/Probability } \\
\cline { 2 - 6 } & $0-1 \%$ & $1-5 \%$ & $5-10 \%$ & $10-50 \%$ & $50-100 \%$ \\
\hline $\begin{array}{c}\text { Production } \\
\text { Shutdown }\end{array}$ & $4 . \mathbf{b}$ & & $\mathbf{1 . c}$ & $\mathbf{1 . e}$ & \\
\hline $\begin{array}{c}\text { Loss of } \\
\text { Product }\end{array}$ & & $\mathbf{3 . a}$ & $\mathbf{6 . b}$ & $\mathbf{2 . b , 5 . e}$ & \\
\hline $\begin{array}{c}\text { Reduced } \\
\text { Speed }\end{array}$ & $\mathbf{1 . b , 1 . f}$ & & & & \\
\hline $\begin{array}{c}\text { Minor } \\
\text { Degradation }\end{array}$ & & & & & \\
\hline $\begin{array}{c}\text { Minor or } \\
\text { No Effect }\end{array}$ & & & & & \\
\hline
\end{tabular}

\begin{tabular}{|c|c|c|c|}
\hline Low & Moderate & High & Extremely \\
Risk & Risk & Risk & High Risk \\
\hline
\end{tabular}

Keeping only the risks that were placed in the "High Risk" and "Extremely High Risk" categories, our new list of scenarios can be seen in Table 7.

Table 7. Reduced list of risk scenarios for Tool Company.

\begin{tabular}{|l|l|l|}
\hline Risk ID & Subsystem & Risk Description \\
\hline $1 . \mathrm{a}$ & Production & Cutoff Saw Failure \\
\hline $1 . \mathrm{c}$ & Production & Lathe Failure \\
\hline $1 . \mathrm{d}$ & Production & Grinder Failure \\
\hline $1 . \mathrm{e}$ & Production & 5-Axis Failure \\
\hline $1 . \mathrm{g}$ & Production & Wrong Tool/Program \\
\hline $2 . \mathrm{b}$ & Engineering & Program Failure \\
\hline $4 . \mathrm{b}$ & Logistics & Missing Tools/Materials \\
\hline 5.e & Management & Missing Human Support \\
\hline
\end{tabular}

\subsection{RFRM Phase 5: Multi-Criteria Evaluation}

The fifth and final phase covered in this modified RFRM methodology is Multi-criteria Evaluation. After identifying the top list of risk scenarios from the previous phases, they must be evaluated by three major criteria: redundancy, resilience, and robustness. Redundancy refers to the ability of extra components or subsystems to take over the functions of failed components or subsystems. Resilience refers to the ability of a system to recover after an emergency, and can be 
evaluated by time and resources needed. Robustness is the insensitivity of system performance to external stresses, so the ability to oppose potential risks (Haimes, 2009).

The three categories of Redundancy, Resilience, and Robustness are then further broken down into eleven essential criteria for evaluating risk scenarios. These are Undetectability, Uncontrollability, Multiple Paths to Failure, Irreversibility, Duration of Effects, Cascading Effects, Operating Environment, Wear and Tear, Interfaces, Complexity \& Emergent Behaviors, and Design Immaturity. However for the PHM-Specific RFRM, we introduce two additional categories: Human and Organizational. These additional categories help to filter out risks that cannot be mitigated through PHM system capabilities. All thirteen criteria for evaluating risk scenarios can be seen in Figure 11.

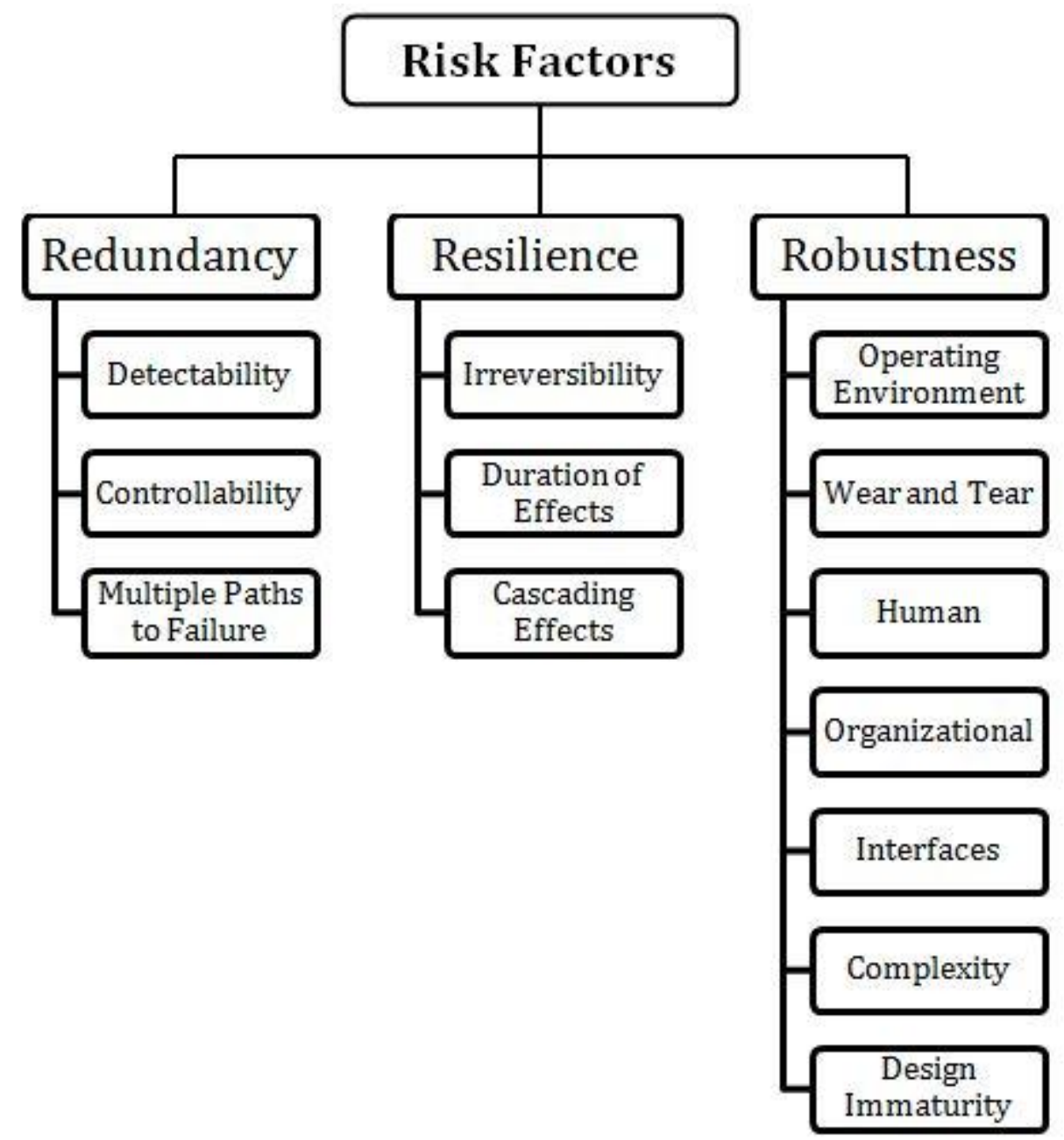

Figure 11. Thirteen criteria to rank risk factors. 
The first eleven criteria relating the ability of a risk scenario to defeat the defenses of a system have been formally defined as follows (Haimes, 2009):

1. Undetectability - The absence of modes by which the initial events of a scenario can be discovered before harm occurs.

2. Uncontrollability - The absence of control modes that make it possible to take action or make an adjustment to prevent harm.

3. Multiple paths to failure - Multiple and possibly unknown ways for the events of a scenario to harm the system.

4. Irreversibility - A scenario in which the adverse condition cannot be returned to the initial, operational (pre-event) condition.

5. Duration of effects - A scenario that would have a long duration of adverse consequences.

6. Cascading effects - A scenario where the effects of an adverse condition propagate to other systems or subsystems (cannot be contained).

7. Operating environment - A scenario that results from external stressors.

8. Wear and tear - A scenario that results from repeated use, leading to degraded performance.

9. Interfaces - A scenario in which the adverse outcome is magnified by interfaces among one or more subsystems (i.e., hardware, software, human, and organizational).

10. Complexity/emergent behaviors - A scenario in which there is a potential for systemlevel behaviors that are not anticipated even with knowledge of components and their interactions.

11. Design immaturity - A scenario in which the adverse consequences are related to the newness of the system design or other lack of a proven concept.

The additional two criteria, which will be used to help filter out risks that cannot be mitigated through PHM system capabilities, have been defined as follows:

12. Human - A scenario in which the adverse outcome is magnified by human error.

13. Organizational - A scenario in which the adverse outcome is magnified by the organizational structure of the facility. 
Each identified risk scenario must be rated as "high", "medium", or "low against each criterion. In general, scenarios with more "high" ratings should be ranked higher in the RFRM process. Risk scenarios that score mostly "low" in the eleven categories can be filtered out unless an emergent change drives it towards a higher level of risk. Alternative rating scales and filtering criteria could also be used in order to reduce the number of scenarios under consideration.

\section{4a PHM-Specific Modifications to RFRM Phase 5}

The Risk Filtering, Ranking, and Management process is essential because it limits the number of risk scenarios for a manufacturing facility to a manageable quantity. However, the process must be modified in order to identify the risks that are applicable to realistic and practical PHM strategies. When scoping for the design of a PHM system, the focus is on failures that result from everyday use of equipment or the passage of time. While outside influences - such as power surges, operator error, or natural disasters - may be the cause of a system failure, PHM addresses failures that occur during normal and intended operation. Therefore, any outside influences must be factored out during the modified RFRM process. Risks that cannot be handled through PHM should still be considered at a higher system level, but will not be useful during the design phase of a PHM system. The modifications to the standard RFRM filtering process are as follows:

M1. The categories Undetectability, Uncontrollability, and Irreversibility are considered to be "tier 1" risks while the other categories are considered to be of "tier 2". Scenarios shall firstly be ranked by number of high, medium, and low ratings in tier 1, and secondly by number of high, medium, and low ratings in tier 2.

M2. A new category shall be added to the list called Human, referring to the effect of human error on the risk. If the risk is completely classified as Human then it shall be filtered out during RFRM.

M3. A new category shall be added to the list called Organizational, referring to the effect of facility's organizational structure on the risk. If the risk is completely classified as Organizational, then it shall be filtered out during RFRM.

M4. Risks that are rated "high" for Undetectability shall be filtered out during RFRM, unless it is possible to add a detection method (such as a sensor to a machine). 
M5. Risks that are rated "high" for Uncontrollability shall be filtered out during RFRM, unless it is possible to insert control modes to the process that could mitigate the risk.

M6. Risks that are directly classified as an Operating Environment risk, and thus cannot be mitigated on a day-to-day basis shall be filtered out during the RFRM.

M7. Risks that are related to hardware, software, or programs that are rated "high" in the category of Design Immaturity and that have never been used, tested, or verified, shall be filtered out during RFRM.

The purpose of the M1 modification is to prioritize the risks with higher ratings in the categories of Undetectability, Uncontrollability, and Irreversibility. These categories are classified as "tier 1 " risk factors because they directly correlate to the overall severity of each risk scenario. The other categories that have been placed in the "tier 2" category are more related to the characteristics and classifications of each risk scenario.

The M2 modification was implemented to help determine the effect of human error on the risk scenario. Risks that are primarily classified as "human" will be eliminated because they are difficult to control and predict with a PHM system. Although it may be beneficial to manage this type of risk, there is little opportunity for PHM to help.

The M3 modification was added to rate the effect of the facility's organizational structure on the risk scenario. Risks that are primarily classified as "organizational" in nature will be eliminated because they are difficult to mitigate with a PHM system. Although it may be beneficial to manage and understand this type of risk, there is little opportunity for PHM.

The purpose of the M4 modification is to ensure that only risks that can be detected, identified, and diagnosed will remain after the filtering process. This is because PHM systems rely on prognostics, and thus require predictive capabilities of future health, performance, or RUL of subsystems. They must have a means to detect or sense in order to provide effective health management. However, it should be noted that if it is possible to add a detection method or even a reliability model to the risk in question, then it should not be filtered out on the basis of the M1 modification. 
The M5 modification seeks to eliminate risks that have no existing control channels. The purpose of a PHM system is to modify decision variables or inputs to a system in order to create a desired outcome. However, even if the optimal modifications to the variables can be identified, if there is no way to implement them, then there is no benefit to the system. It was additionally noted that if it is possible to add control modes, then this filtering criterion can be ignored.

The purpose of the M6 modification is to filter out risks that are only related to the operating environment. Specifically, these are the risks pertaining to external factors over which there is no control, such as the weather, plant location, and even legislation or industry standards. These risks should be filtered because they cannot be managed on a day-to-day basis and would require solutions outside the scope of a manufacturing PHM system. It should be noted that this should only serve as a filter if it is the only "high" rated risk category.

Finally, the M7 modification removes risks that are focused on immature or experimental subsystems, which are usually still undergoing optimization or usability testing. These new systems will naturally inherit additional risk since they have not yet been verified. Therefore, we would not want to allocate resources towards developing a PHM system for a new component until it has become stable within its own design cycle.

\section{4b RFRM Phase 5 Flowcharts}

In order to assist the analyst with the RFRM process, flowcharts have been developed outlining how to rate each scenario based on thirteen essential criteria. The developed flowcharts provide a repeatable methodology to assign ratings through a series of objective questions. The benefit of the flowcharts is that they do not require a risk analyst or systems engineer in order for a manufacturing company to prioritize their risk scenarios. The top level RFRM flowchart provides a map for the entire rating process (refer to Figure 12). 


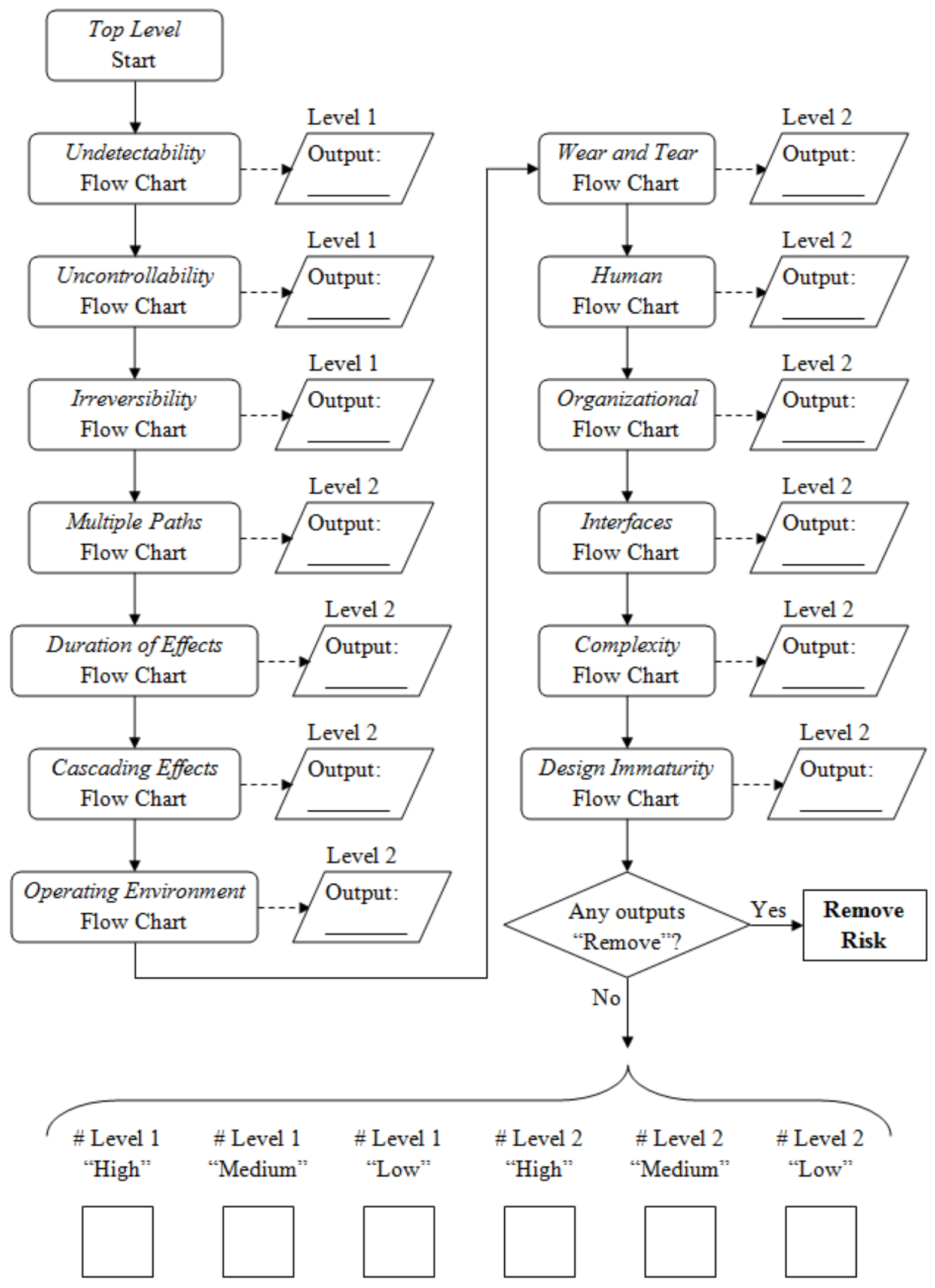

Figure 12. Top Level RFRM Chart. 
This top level RFRM flowchart incorporates all of the PHM-specific modifications M1 through M7. It also breaks down the rating of each of the thirteen essential criteria into their own individual sub flowcharts. It can be seen that the outcome of the RFRM process is a 6-digit number corresponding to the number of high/medium/low ratings for the tier 1 categories, and the number of high/medium/low ratings for the tier 2 categories. The risk scenarios with higher 6-digit outputs should be ranked higher on an ordinal scale than scenarios with lower outputs. The support flowcharts for the thirteen risk criteria that are referenced in the top level flowchart can be found in Appendix D. Support Flowcharts for RFRM Phase 5 Multi-Criteria Evaluation.

\section{4c Food Company - Multi-Criteria Evaluation}

Following the RFRM methodology, a multi-criteria evaluation was performed on the top risk scenarios identified in the Food Company case study. The thirteen different risk categories received a qualitative rating of either "high", "medium", "low", or "remove" by following the prompts and questions in the developed flowcharts. Table 8 shows the full multi-criteria evaluation analysis with all of the qualitative ratings. Table 9 shows the number of "high", "medium", and "low" ratings for Level 1 and Level 2 categories, as well as any scenarios that received a "remove" rating. Finally, Table 10 lists the risk scenarios in their rank order of priority for receiving PHM system capabilities. 
Table 8. Food Company Multi-Criteria Evaluation.

\begin{tabular}{|c|c|c|c|c|c|c|c|c|c|c|c|c|c|c|}
\hline Risk ID & Risk Scenario & 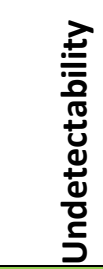 & 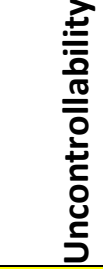 & 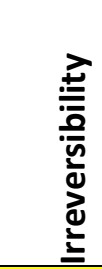 & 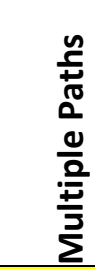 & 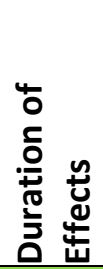 & 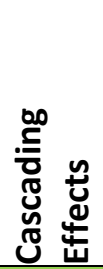 & 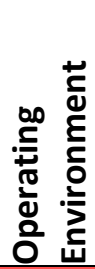 & 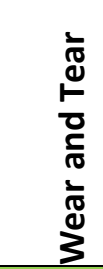 & 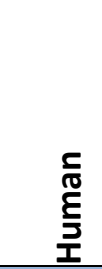 & 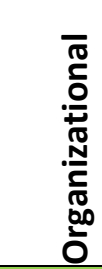 & 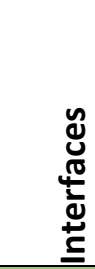 & $\frac{\frac{\lambda}{x}}{\frac{\pi}{0}}$ & 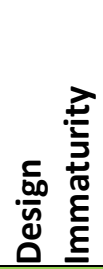 \\
\hline 3.a & Human Operator Failure & Low & Med & Med & Med & Low & Low & High & Low & Rem. & Low & Low & Low & Low \\
\hline 3.b & Bagging Machine Failure & Med & Low & Med & Med & Low & Med & Med & Med & High & Low & Med & Low & Low \\
\hline 6.b & Flattening Machine Failure & Med & Low & Low & Low & Med & Med & Low & Med & Low & Low & Low & Low & Low \\
\hline 7.c & Palletizing Robot Failure & High & Low & High & Med & Med & High & Med & Med & Low & Low & High & Med & Med \\
\hline
\end{tabular}

Table 9. Food Company Multi-Criteria Evaluation Numbers.

\begin{tabular}{|c|l|c|c|c|c|c|c|c|}
\hline Risk ID & Risk Scenario & $\begin{array}{c}\text { Level 1 } \\
\text { High }\end{array}$ & $\begin{array}{c}\text { Level 1 } \\
\text { Medium }\end{array}$ & $\begin{array}{c}\text { Level 1 } \\
\text { Low }\end{array}$ & $\begin{array}{c}\text { Level 2 } \\
\text { High }\end{array}$ & $\begin{array}{c}\text { Level 2 } \\
\text { Medium }\end{array}$ & $\begin{array}{c}\text { Level 2 } \\
\text { Low }\end{array}$ & $\begin{array}{c}\text { Remove } \\
\text { Risk? }\end{array}$ \\
\hline 3.a & Human Operator Failure & 0 & 2 & 1 & 1 & 1 & 7 & Yes \\
\hline 3.b & Bagging Machine Failure & 0 & 2 & 1 & 1 & 5 & 4 & No \\
\hline 6.b & Flattening Machine Failure & 0 & 1 & 2 & 0 & 3 & 7 & No \\
\hline 7.c & Palletizing Robot Failure & 2 & 0 & 1 & 2 & 6 & 2 & No \\
\hline
\end{tabular}

Table 10. Rank Order of Food Company Risk Scenarios.

\begin{tabular}{|c|c|l|}
\hline Rank & Risk ID & Risk Scenario \\
\hline 1 & $7 . c$ & Palletizing Robot Failure \\
\hline 2 & $3 . b$ & Bagging Machine Failure \\
\hline 3 & $6 . b$ & Flattening Machine Failure \\
\hline $\mathbf{X}$ & $3 . \mathrm{a}$ & Human Operator Failure \\
\hline
\end{tabular}


From Table 10, we find that the top risk scenario remaining under consideration for Food Company is the Palletizing Robot Failure. This scenario received the most "High" ratings among the Level 1 criteria. The Bagging Machine Failure came in second place with the most "Medium" ratings in Level 1 criteria, followed closely by the Flattening Machine Failure. The scenario titled Human Operator Failure was removed from consideration since it received a "Remove" rating in the criteria category labeled "Human".

Given limited resources, it is recommended that the Food Company focus their PHM strategy efforts at the Palletizer Robot. This will provide the biggest impact towards achieving their objectives: maximize production and minimize the risk of a system failure (production shutdown or delay). A Fault Tree Analysis (discussed in the next section) can provide further guidance to determine which components of the Palletizer Robot are most critical. Then if the Food Company would like to continue implementing PHM systems and solutions, they should prioritize the Bagging Machine subsystem second, and the Flattening Machine subsystem third.

\section{4d Tool Company - Multi-Criteria Evaluation}

The Multi-Criteria Evaluation phase was also performed on the top risk scenarios identified in the Tool Company case study. The thirteen different risk categories received a qualitative rating of either "high", "medium", "low", or "remove" by following the prompts and questions in the developed flowcharts. Table 11 shows the full multi-criteria evaluation analysis with all of the qualitative ratings. Table 12 shows the number of "high", "medium", and "low" ratings for Level 1 and Level 2 categories, as well as any scenarios that received a "remove" rating. Finally, Table 13 lists the risk scenarios in their rank order of priority for receiving PHM system capabilities. 
Table 11. Tool Company Multi-Criteria Evaluation.

\begin{tabular}{|c|c|c|c|c|c|c|c|c|c|c|c|c|c|c|}
\hline Risk ID & Risk Scenario & 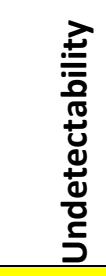 & 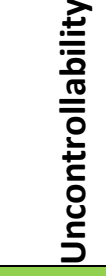 & 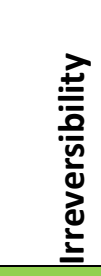 & $\begin{array}{l}\frac{n}{1} \\
\frac{1}{\pi} \\
\frac{0}{2} \\
\frac{2}{2} \\
\frac{2}{2}\end{array}$ & 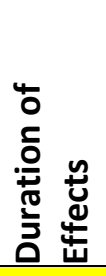 & 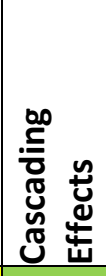 & 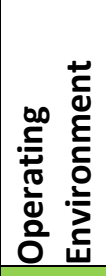 & 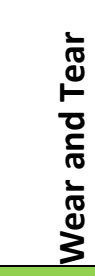 & $\begin{array}{l}\frac{5}{0} \\
\frac{\delta}{2} \\
\text { I }\end{array}$ & 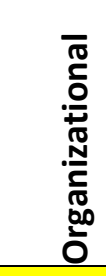 & 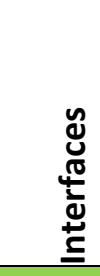 & $\frac{\stackrel{\nexists}{x}}{\frac{0}{0}}$ & 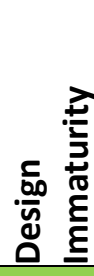 \\
\hline 1.a & Cutoff Saw Failure & Med & Low & Low & High & Med & Low & Low & Low & High & Med & Low & Med & Low \\
\hline 1.c & Lathe Failure & High & Med & High & Low & Low & Low & Low & Med & Med & Low & Low & Low & Low \\
\hline 1.d & Grinder Failure & High & Med & Med & Low & Med & Low & Low & High & Low & Low & Low & Low & Low \\
\hline 1.e & 5-Axis Failure & High & High & High & Low & Med & Low & Low & High & Low & Low & Low & Low & Low \\
\hline 1.g & Wrong Tool/Program & High & Med & Med & Low & Low & Low & Low & Low & Rem. & Low & Low & Low & Low \\
\hline 2.b & Program Failure & Med & Low & Low & High & Low & Low & Low & Low & Med & Med & Med & Med & Low \\
\hline 4.b & Missing Tools/Materials & Low & Rem. & Med & High & High & Med & Med & Low & Rem. & Med & Med & Med & Low \\
\hline 5.e & Missing Human Support & Low & Low & Med & Low & Med & Med & Low & Low & Low & Rem. & Med & Med & Low \\
\hline
\end{tabular}

Table 12. Tool Company Multi-Criteria Evaluation Numbers.

\begin{tabular}{|c|l|c|c|c|c|c|c|c|}
\hline Risk ID & Risk Scenario & $\begin{array}{c}\text { Level 1 } \\
\text { High }\end{array}$ & $\begin{array}{c}\text { Level 1 } \\
\text { Medium }\end{array}$ & $\begin{array}{c}\text { Level 1 } \\
\text { Low }\end{array}$ & $\begin{array}{c}\text { Level 2 } \\
\text { High }\end{array}$ & $\begin{array}{c}\text { Level 2 } \\
\text { Medium }\end{array}$ & $\begin{array}{c}\text { Level 2 } \\
\text { Low }\end{array}$ & $\begin{array}{c}\text { Remove } \\
\text { Risk? }\end{array}$ \\
\hline 1.a & Cutoff Saw Failure & 0 & 1 & 2 & 2 & 3 & 5 & No \\
\hline 1.c & Lathe Failure & 2 & 1 & 0 & 0 & 2 & 8 & No \\
\hline 1.d & Grinder Failure & 1 & 2 & 0 & 1 & 1 & 8 & No \\
\hline 1.e & 5-Axis Failure & 3 & 0 & 0 & 1 & 1 & 8 & No \\
\hline 1.g & Wrong Tool/Program & 1 & 2 & 0 & 0 & 0 & 9 & Yes \\
\hline 2.b & Program Failure & 0 & 1 & 2 & 1 & 4 & 5 & No \\
\hline 4.b & Missing Tools/Materials & 0 & 1 & 1 & 2 & 5 & 2 & Yes \\
\hline 5.e & Missing Human Support & 0 & 1 & 2 & 0 & 4 & 5 & Yes \\
\hline
\end{tabular}


Table 13. Rank Order of Tool Company Risk Scenarios.

\begin{tabular}{|c|c|l|}
\hline Rank & Risk ID & Risk Scenario \\
\hline 1 & 1.e & 5-Axis Failure \\
\hline 2 & $1 . c$ & Lathe Failure \\
\hline 3 & $1 . \mathrm{d}$ & Grinder Failure \\
\hline 4 & $1 . \mathrm{a}$ & Cutoff Saw Failure \\
\hline 5 & $2 . \mathrm{b}$ & Program Failure \\
\hline $\mathbf{X}$ & 1.g & Wrong Tool/Program \\
\hline $\mathbf{X}$ & 4.b & Missing Tools/Materials \\
\hline $\mathbf{X}$ & 5.e & Missing Human Support \\
\hline
\end{tabular}

From Table 13, we find that the top risk scenario remaining under consideration for Tool Company is the 5-Axis Failure. This scenario received the most "High" ratings among the Level 1 criteria. After this scenario came Lathe Failure, Grinder Failure, Cutoff Saw Failure, and finally Program Failure. The scenarios Wrong Tool/Program, Missing Tools/Materials, and Missing Human Support were removed from consideration because they received one or more "Remove" ratings within the RFRM flowcharts. Scenario 1.g (wrong tool/program) was classified as human error, scenario 4.b (missing tools/materials) was considered to be uncontrollable as well as human error, and scenario 5.e (missing human support) was classified as an organizational issue.

Given limited resources, it is recommended that the Tool Company focus their PHM strategy efforts at the 5-Axis Machine. This will provide the biggest impact towards achieving their major objective of maximizing production. A Fault Tree Analysis (discussed in the next section) can provide further guidance to determine which components of the 5-Axis Machine are most critical. Then if the Tool Company would like to continue implementing PHM systems and solutions, they should continue down the list displayed in Table 13. 


\section{Fault Tree Analysis}

Fault Tree Analysis (FTA) is the final step in the risk analysis methodology that provides scope for a PHM system designer. It should be used after the identification of high-priority PHM subsystems from the HHM, RFRM, and PHM-specific RFRM methodologies. Fault Tree Analysis helps to determine exactly how a risk scenario could occur, and more specifically which components might be involved in the failure of a manufacturing machine. The expected result of FTA is a list of the smallest combination of component failures that would lead to a subsystem failure. This combination is known in risk analysis as a minimal cut set. A single failure from any of the components in the minimal cut set will propagate to failure of the top event (Haimes, 2012). A PHM system designer can use knowledge of these components to provide guidance for locations of sensors or health monitoring devices.

A fault tree is created using the components shown in Figure 13. The top event represents the risk scenario of interest, which was determined from the RFRM methodology in the previous section. This top event then breaks down into multiple levels of intermediate events, until finally ending at the bottom with an undeveloped event. In theory, these undeveloped events should represent components in a machine hierarchy which would most benefit from PHM. The events in a fault tree are connected by AND gates and OR gates. If events are connected with AND gates, they are essentially in parallel and all of the events must occur in order for the above event to trigger. If events are connected with OR gates, they are essentially in series so the occurrence of any of the events would cause the above event to trigger. 


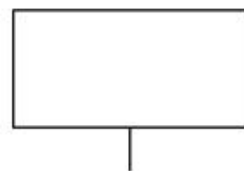

Top Event: The primary undesired event of interest for fault tree analysis.

Denoted by a rectangle.

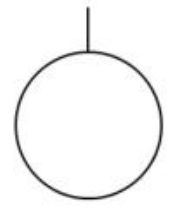

Basic Event: An event which requires no further development

Denoted by a circle.

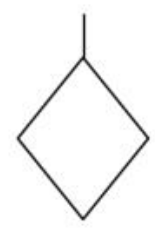

Undeveloped Event: Another event which is not developed further, either because it is low consequence or because relevant information is not available Denoted by a diamond.

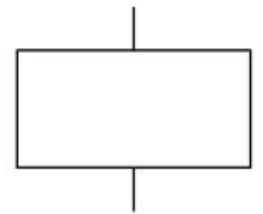

Intermediate Event: The fault event which is developed further. Denoted by a rectangle.

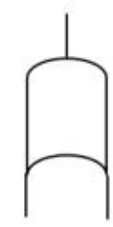

OR Gate: Shows that the output event occurs only if one or more of the input events occur. There can be any number of inputs to an OR gate

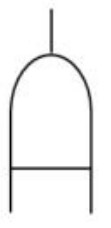

AMD Gate: Shows that the output fault event occurs if, and only if, all the input events occur. There can be any number of inputs to an AND gate.

\section{Figure 13. Fault Tree Analysis components.}

The minimal cut set of a fault tree is calculated using Boolean algebra. Since OR gates are in series, their events must be added together (in union) in order for the top event to occur:

$$
\mathrm{T}_{-} \mathrm{OR}=\mathrm{E}_{1}+\mathrm{E}_{2}
$$

Since AND gates are in parallel, their events are combined (in intersection) with a multiplication symbol:

$$
\mathrm{T}_{\text {AND }}=\mathrm{E}_{1} \bullet \mathrm{E}_{2} \quad \text { or } \quad \mathrm{T}_{\_\mathrm{AND}}=\mathrm{E}_{1} \mathrm{E}_{2}
$$

Following the above logic, the top event in a fault tree becomes an expression combining the Undeveloped Events of the tree. This expression can then be reduced using the laws of set algebra (e.g., Associative Law, Commutative Law, and Distributive Law).

In order to facilitate the Fault Tree Analysis in the context of PHM, the analyst should refer to the hierarchy of risk scenarios developed in the RFRM methodology. These scenarios should have been created from a production perspective, so their aspects will represent components of the machine/subsystem of interest. The two case studies with Food Company and Tool Company provide examples of this process. 


\subsection{Food Company Fault Tree Analysis}

Since the top risk scenario from the RFRM methodology was the Palletizing Robot Failure, this was used as the top event for the Fault Tree Analysis. The fault tree for Food Company can be seen in Figure 14.

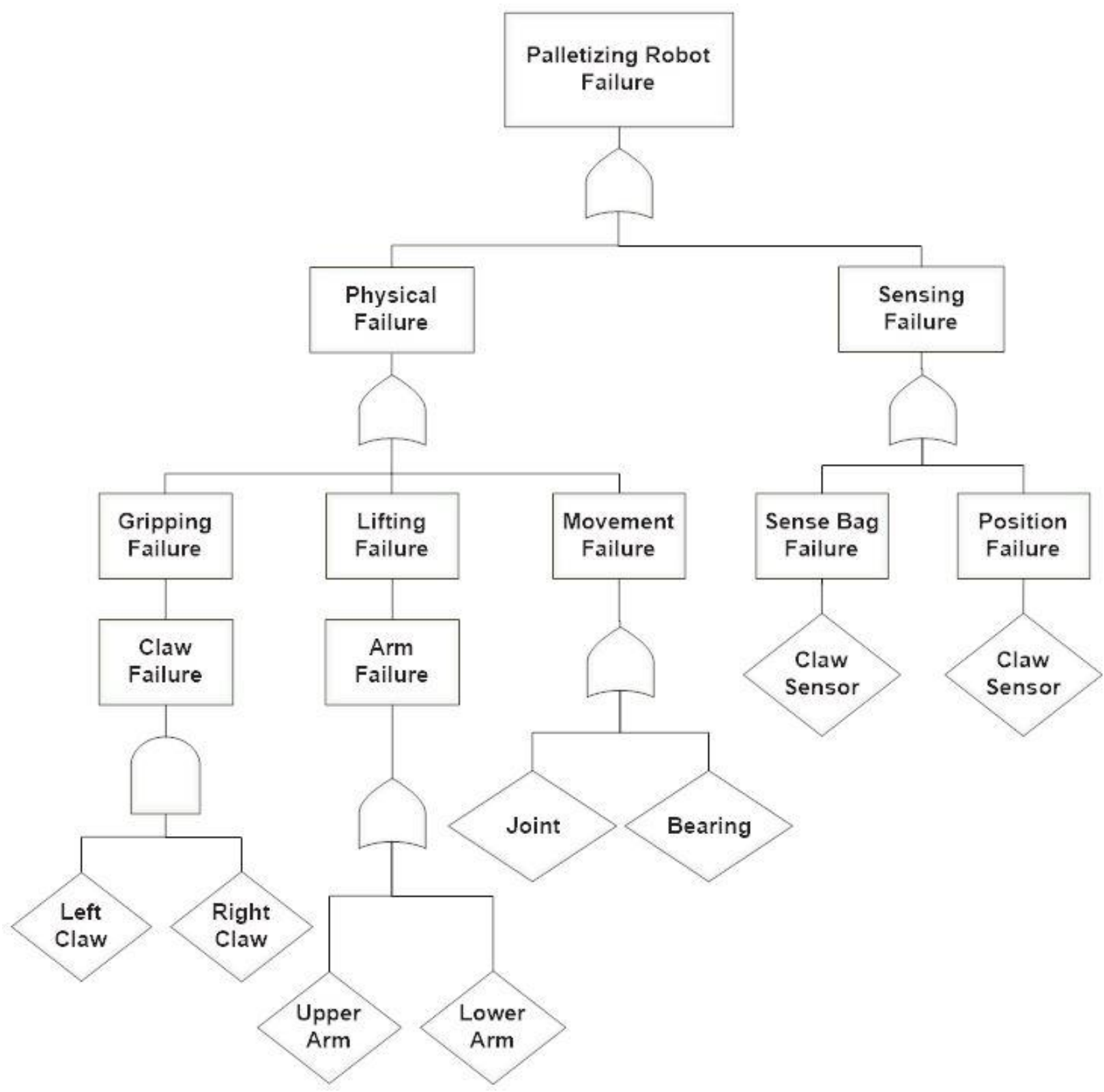

Figure 14. Food Company - Palletizing Robot Failure Fault Tree. 
In order to simplify the equations for calculating the minimal cut set, the following notations were used to represent the fault tree events:

T: Palletizing Robot Failure

$\mathrm{A}_{1}$ : $\quad$ Physical Failure

$\mathrm{A}_{21}$ : Gripping Failure / Claw

Failure

$\mathrm{A}_{22}$ : Lifting Failure / Arm Failure

$\mathrm{A}_{23}$ : Movement Failure

$\mathrm{A}_{31}$ : Left Claw

$\mathrm{A}_{32}$ : Right Claw
$\mathrm{A}_{33}$ : Upper Arm

$\mathrm{A}_{34}: \quad$ Lower Arm

A 35 : Joint

$\mathrm{A}_{36}$ : Bearing

$\mathrm{B}_{1}$ : $\quad$ Sensing Failure

$\mathrm{B}_{21}$ : Sense Bag Failure

$\mathrm{B}_{22}$ : Position Failure

$\mathrm{B}_{31}$ : Claw Sensor

$$
\begin{array}{ll}
\mathrm{T}= & \mathrm{A}_{1}+\mathrm{B}_{1} \\
\mathrm{~T}= & \mathrm{A}_{21}+\mathrm{A}_{22}+\mathrm{A}_{23}+\mathrm{B}_{21}+\mathrm{B}_{22} \\
\mathrm{~T}= & \mathrm{A}_{31} \mathrm{~A}_{32}+\mathrm{A}_{33}+\mathrm{A}_{34}+\mathrm{A}_{35}+\mathrm{A}_{36}+\mathrm{B}_{31}+\mathrm{B}_{31} \\
\mathrm{~T}= & \mathrm{A}_{31} \mathrm{~A}_{32}+\mathrm{A}_{33}+\mathrm{A}_{34}+\mathrm{A}_{35}+\mathrm{A}_{36}+\mathrm{B}_{31}
\end{array}
$$

From the calculations above, the minimal cut set for the Palletizing Robot Failure is comprised of the following component failures:

\section{Left Claw \& Right Claw}

2. Upper Arm

3. Lower Arm

\section{Joint}

\section{Bearing}

\section{Claw Sensor}

These six components all represent viable targets for PHM sensors within the Palletizing Robot. 


\subsection{Tool Company Fault Tree Analysis}

The top risk scenario from the RFRM methodology with the Tool Company was the 5-Axis Machine Failure. This scenario was used as the top event for the Fault Tree Analysis, which can be seen in Figure 15.

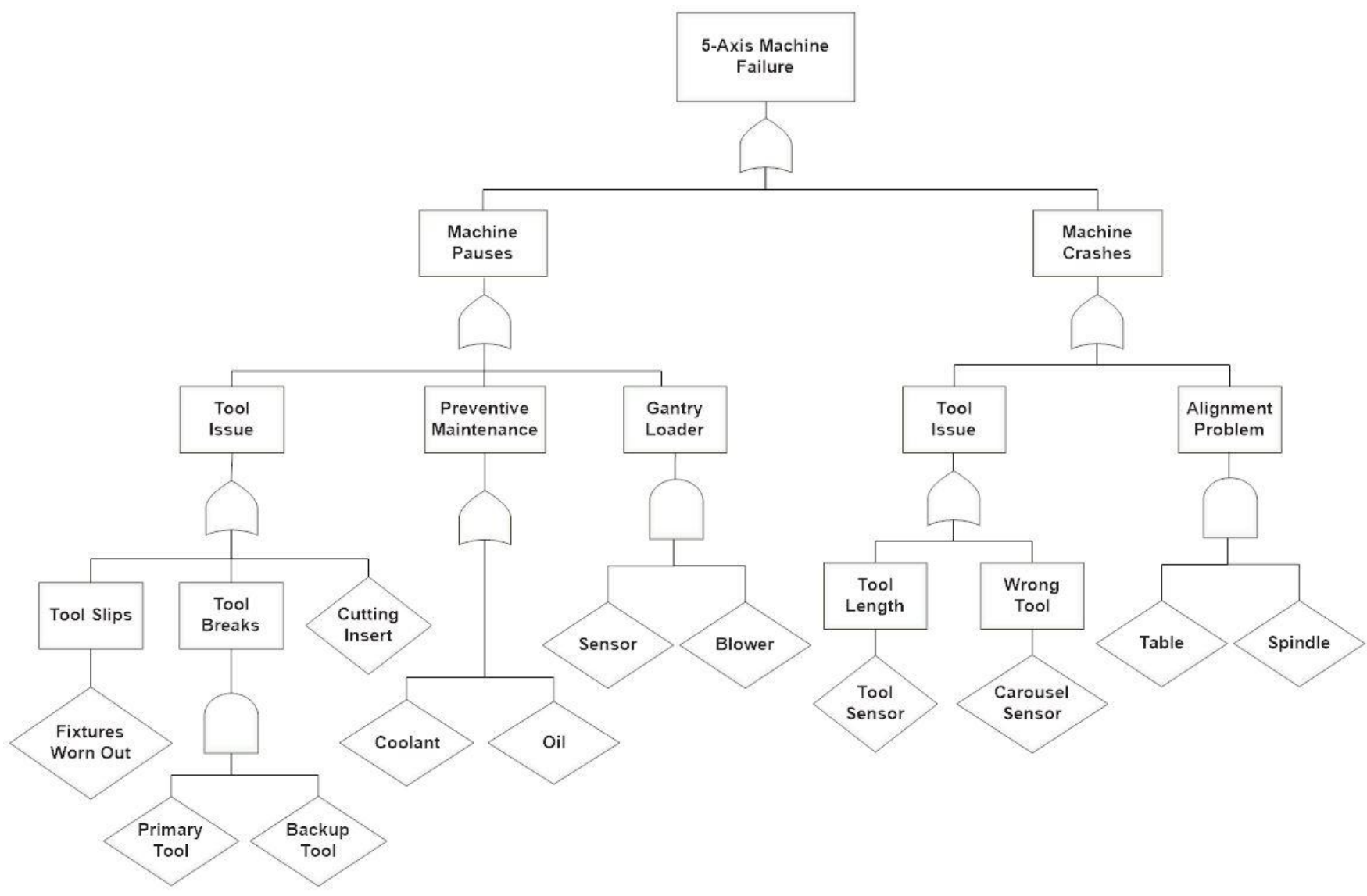

Figure 15. Tool Company - 5-Axis Machine Failure Fault Tree.

In order to simplify the equations for calculating the minimal cut set, the following notations were used to represent the fault tree events:

T: $\quad$ 5-Axis Machine Failure

$\mathrm{A}_{1}$ : Machine Pauses

$\mathrm{A}_{21}$ : Tool Issue

$\mathrm{A}_{22}$ : Preventive Maintenance

$\mathrm{A}_{23}$ : Gantry Loader

$\mathrm{A}_{31}$ : Tool Slips
$\mathrm{A}_{32}$ : Tool Breaks

$\mathrm{A}_{33}$ : Cutting Insert

$\mathrm{A}_{34}$ : Coolant

$\mathrm{A}_{35}$ : Oil

$\mathrm{A}_{36}$ : Gantry Loader Sensor

A 37 Gantry Loader Blower 

$\mathrm{A}_{41}$ : Fixtures Worn Out
$\mathrm{B}_{31}$ : Tool Length
$\mathrm{A}_{42}$ : Primary Tool
$\mathrm{B}_{32}$ ： Wrong Tool
$\mathrm{A}_{43}$ : Backup Tool
$\mathrm{B}_{33}$ : Table
$\mathrm{B}_{1}$ : Machine Crashes
$\mathrm{B}_{34}: \quad$ Spindle
$\mathrm{B}_{21}$ : Tool Issue
$B_{41}$ : Tool Sensor
$\mathrm{B}_{22}$ : Alignment Problem
$\mathrm{B}_{42}$ : Carousel Sensor

$\mathrm{T}=\quad \mathrm{A}_{1}+\mathrm{B}_{1}$

$\mathrm{T}=\mathrm{A}_{21}+\mathrm{A}_{22}+\mathrm{A}_{23}+\mathrm{B}_{21}+\mathrm{B}_{22}$

$\mathrm{T}=\mathrm{A}_{31}+\mathrm{A}_{32}+\mathrm{A}_{33}+\mathrm{A}_{34}+\mathrm{A}_{35}+\mathrm{A}_{36} \mathrm{~A}_{37}+\mathrm{B}_{31}+\mathrm{B}_{32}+\mathrm{B}_{33} \mathrm{~B}_{34}$

$\mathrm{T}=\mathrm{A}_{41}+\mathrm{A}_{42} \mathrm{~A}_{43}+\mathrm{A}_{33}+\mathrm{A}_{34}+\mathrm{A}_{35}+\mathrm{A}_{36} \mathrm{~A}_{37}+\mathrm{B}_{41}+\mathrm{B}_{42}+\mathrm{B}_{33} \mathrm{~B}_{34}$

From the calculations above, the minimal cut set for the 5-Axis Machine Failure is comprised of the following component failures:

\section{Fixtures}

2. Primary Tool \& Backup Tool

3. Cutting Insert

4. Coolant

5. Oil

6. Gantry Loader Sensor \& Gantry Loader Blower

7. Tool Sensor

8. Carousel Sensor

9. Table \& Spindle

These nine components all represent viable targets for PHM sensors within the 5-Axis Machine. 


\section{Part V: Summary and Conclusions}

\section{Major Findings and Contributions}

The major findings from this thesis were the results from the case studies with the two anonymous manufacturing companies. With the Food Company, it was determined that the top risk scenarios were the Palletizing Robot Failure, the Bagging Machine Failure, and the Flattening Machine Failure. From the fault tree analysis on the Palletizing Robot Failure, the major components that would most benefit from PHM were: (1) Left/Right Claws, (2) Upper Arm, (3) Lower arm, (4) Joint, (5) Bearing, and (6) Claw Sensor. For the Tool Company, the top risk scenarios were the 5-Axis Failure, Lathe Failure, Grinder Failure, Cutoff Saw Failure, and Program Failure. Again, a fault tree analysis was conducted for the top-rated risk scenario. It was determined that the most critical components of the 5-Axis Machine were: (1) Fixtures, (2) Primary/Backup Tool, (3) Cutting Insert, (4) Coolant, (5) Oil, (6) Gantry Loader Sensor/Blower, (7) Tool Sensor, (8) Carousel Sensor, and (9) Table/Spindle. These results were presented to the decision makers at their respective companies, who confirmed that they would use the information when scoping/designing a PHM system and developing their risk management strategies.

A major contribution of this thesis is a methodology that can be used by manufacturing companies to identify the major targets that would most benefit from a PHM system. The field of Risk Analysis (modeling, assessment, management, and communication) provided systematic and well-established approaches to identifying the major risk scenarios that would impair a system from achieving its major objectives. However, the methodologies required modifications so that they could be applicable and relevant for a manufacturing process seeking PHM capabilities. The developed methodology integrated Hierarchical Holographic Modeling (HHM), Risk Filtering, Ranking, and Management (RFRM), and Fault Tree Analysis (FTA) to provide scope and direction for a PHM system designer.

Another contribution of this thesis was the development of the Risk in Manufacturing Processes Survey. This survey provides a huge benefit to the Hierarchical Holographic Modeling methodology by facilitating the integration of expert knowledge without requiring the stakeholders to collaborate simultaneously and in person. It also provides a very repeatable 
process that any manufacturer could replicate. In a similar vein, the developed flowcharts for the multi-criteria risk evaluation also benefit the overall RFRM process. The flowcharts provide a systematic series of questions that a decision maker or stakeholder can follow to rate and filter their risk scenarios.

The final major contribution of this thesis was the merging and harmonization of the academic fields of Risk Analysis with Prognostics and Health Management. Risk Analysis provides many well-established tools, resources, methodologies, and theories that can advance the growing field of Prognostics and Health Management. In order to justify using these tools within a PHM context, I demonstrated that the two practices were in fact compatible. The similarities between Risk Analysis and PHM were solidified through an investigation and comparison between the Dynamic Roadmap for Risk Modeling, Planning, Assessment, Management, and Communication with the Major Design Components of Prognostics and Health Management. The benefits to PHM from implementing Risk Analysis methodology were further outlined through the two case studies with U.S. manufacturing industry.

\section{Key Challenges}

A major challenge of this thesis was determining how to adapt the risk analysis methodologies towards designing and scoping a PHM system, and then justifying the modifications. The secondary objective of this thesis was therefore developed to help with the justification; by first harmonizing the fields of Risk Analysis and Prognostics and Health Management, I was able to rationalize using the HHM, RFRM, and FTA methodologies.

Another challenge of this research was coordinating with the managers, stakeholders, and decision makers at the two separate manufacturing industries. It was especially difficult since the facilities were quite different in size, organizational structure, and products. The Risk in Manufacturing Processes Survey and the RFRM flowcharts were therefore developed to ease the collaboration efforts and integrate as much expert knowledge as possible into the models. 


\section{Recommendations for Future Work}

The research presented in this thesis is part one of a NIST Measurement Science and Engineering Research Grant project titled Methodologies for Real-Time Diagnostics and Prognostics in Smart Manufacturing Systems. The purpose of this first part was to provide scope to a PHM system designer, by identifying targets within a manufacturing environment that would most benefit from such a system. The second part, which is being led by another member of my research team, is to design and simulate a PHM system using real maintenance data from the two manufacturing companies discussed in this paper. Finally, the third part of this effort is to provide decision support and risk management to the identified companies by comparing the tradeoffs between different maintenance and operational strategies.

Besides the second and third parts of this research discussed above, this project would greatly benefit from additional case studies to further refine and verify the methodology. It would also help to measure the actual production, cost, or health benefits achieved by the companies after developing and implementing their PHM systems. Finally, since this thesis proposes the idea that Risk Analysis and Prognostics and Health Management are harmonious and compatible, future projects could involve additional collaboration efforts towards adapting and modifying methodologies between these two fields. 


\section{References}

Ahmad, R., \& Kamaruddin, S. (2012). An overview of time-based and condition-based maintenance in industrial application. Computers \& Industrial Engineering, vol. 63 (1), pp. 135-149.

Al-Habaibeh, A., \& Gindy, N. (2000). A new approach for systematic design of condition monitoring systems for milling processes. Journal of Materials Processing Technology, vol. 107 (1), pp. 243-251.

Alvandi, S., Bienert, G., Li, W., \& Kara, S. (2015). Hierarchical modelling of complex material and energy flow in manufacturing systems. Procedia CIRP, vol. 29, pp. 92-97.

Andretta, M. (2014). Some considerations on the definition of risk based on concepts of systems theory and probability. Risk Analysis, vol. 34 (7), pp. 1184-1195.

Bai, G., Wang, P., \& Hu, C. (2015). A self-cognizant dynamic system approach for prognostics and health management. Journal of Power Sources, vol. 278, pp. 163-174. doi:10.1016/j.jpowsour.2014.12.050.

Barajas, L. G., \& Srinivasa, N. (2008). Real-time diagnostics, prognostics and health management for large-scale manufacturing maintenance systems. ASME 2008 International Manufacturing Science and Engineering Conference collocated with the 3rd JSME/ASME International Conference on Materials and Processing (pp. 85-94). American Society of Mechanical Engineers.

Batzel, T. D., \& Swanson, D. C. (2009). Prognostic health management of aircraft power generators. In IEEE Transactions on Aerospace and Electronic Systems, vol. 45 (2), pp. 473482.

Biehl, S., Staufenbiel, S., Recknagel, S., Denkena, B., \& Bertram, O. (2012). Thin film sensors for condition monitoring in ball screw drives. $1^{\text {st }}$ Joint International Symposium on SystemIntegrated Intelligence: New Challenges for Product and Production Engineering.

Chand, S., \& Davis, J. F. (2010). What is smart manufacturing. Time Magazine Wrapper, 28-33.

Chittester, C. G., \& Haimes, Y. Y. (2004). Risks of terrorism to information technology and to critical interdependent infrastructures. Journal of Homeland Security and Emergency Management, vol. 1 (4).

Cocheteux, P., Voisin, A., Levrat, E., Iung, B. (2009). Prognostic design: requirements and tools. $11^{\text {th }}$ International Conference on The Modern Information Technology in the Innovation Processes of the Industrial Enterprises, MITIP 2009. Bergame, Italy.

Crowther, K. G., Dicdican, R. Y., Leung, M. F. , Lian, C., \& Williams, G. M. (2004). Assessing and managing risk of terrorism to Virginia's interdependent transportation systems. Center for Risk Management of Engineering Systems, Charlottesville, Virginia.

Dombroski, M., Haimes, Y. Y., Lambert, J. H., Schlussel, K., \& Sulcoski, M. (2002). Risk-based methodology for support of operations other than war. Military Operations Research, vol. 7 (1), pp. 19-38.

Feldman, K., Jazouli, T., \& Sandborn, P. (2009). A methodology for determining the return on investment associated with prognostics and health management. IEEE Transactions on Reliability, vol. 58 (2), pp. 305-316.

Fernández, F. B., \& Pérez, M. Á. S. (2015). Analysis and modeling of new and emerging occupational risks in the context of advanced manufacturing processes. Procedia Engineering, vol. 100, pp. 1150-1159. 
Haimes, Y. Y. ( ${ }^{\text {rd }}$ ed.). (2009). Risk modeling, assessment, and management. Hoboken, NJ: John Wiley \& Sons, Inc.

Haimes, Y. Y. (2012). Systems-based guiding principles for risk modeling, planning, assessment, management, and communication. Risk Analysis, vol. 32 (9), pp. 1451-1467.

Haimes, Y. Y. (4 ${ }^{\text {th }}$ ed.). (2016). Risk modeling, assessment, and management. Hoboken, NJ: John Wiley \& Sons, Inc.

Haimes, Y. Y., \& Horowitz, B. M. (2004). Adaptive two-player hierarchical holographic modeling game for counterterrorism intelligence analysis. Journal of Homeland Security and Emergency Management, vol. 1 (3).

Haimes, Y. Y., Kaplan, S., \& Lambert, J. H. (2002). Risk filtering, ranking, and management framework using hierarchical holographic modeling. Risk Analysis, vol. 22 (2), pp. 383-397. doi:10.1111/0272-4332.00020.

He, N., Zhang, D. Z., \& Li, Q. (2014). Agent-based hierarchical production planning and scheduling in make-to-order manufacturing system. International Journal of Production Economics, vol. 149, pp. 117-130.

Hou-bo, H., \& Jian-min, Z. (2011). Cost-benefit model for PHM. Procedia Environmental Sciences, vol. 10, pp. 759-764.

Hofmeister, J. P., Wagoner, R. S., \& Goodman, D. L. (2013). Prognostic health management (PHM) of electrical systems using condition-based data for anomaly and prognostic reasoning. Italian Association of Chemical Engineering, vol. 33, pp. 991-996. doi: 10.3303/CET1333166.

Holland, S. W., Barajas, L. G., Salman, M., \& Zhang, Y. (2010). PHM for automotive manufacturing \& vehicle applications. Prognostics \& Health Management Conference Fielded Systems Session, October 14, Portland, Oregon.

Jung K., Morris K. C., Lyons K. W., Leong S., \& Cho H. (2015). Mapping strategic goals and operational performance metrics for smart manufacturing systems. Procedia Computer Science, vol. 44, pp. 184-193. doi:10.1016/j.procs.2015.03.051.

Kaplan, S., Garrick, B. (1981). On the quantitative definition of risk. Risk Analysis, vol. 1 (1), pp. 11-27.

Kaplan, S., Haimes, Y. Y., \& Garrick, B. J. (2001). Fitting hierarchical holographic modeling into the theory of scenario structuring and a resulting refinement to the quantitative definition of risk. Risk Analysis, vol. 21 (5), pp. 807-807.

Lambert, J. H., Haimes, Y. Y., Li, D., Schooff, R. M., \& Tulsiani, V. (2001). Identification, ranking, and management of risks in a major system acquisition. Reliability Engineering \& System Safety, vol. 72 (3), pp. 315-325.

Lee, C. K. M., Lv, Y., \& Hong, Z. (2013). Risk modelling and assessment for distributed manufacturing system. International Journal of Production Research, vol. 51 (9), pp. 26522666.

Lee, J., Wu, F., Zhao, W., Ghaffari, M., Liao, L., \& Siegel, D. (2014). Prognostics and health management design for rotary machinery systems - Reviews, methodology and applications. Mechanical Systems and Signal Processing, vol. 42 (1-2), pp. 314-334. doi:10.1016/j.ymssp.2013.06.004.

Malinowski, M. L., Beling, P. A., Haimes, Y. Y., LaViers, A., Marvel, J. A., \& Weiss, B. A. (2015). System Interdependency Modeling in the Design of Prognostic and Health Management Systems in Smart Manufacturing. 
National Institute of Standards and Technology (NIST). (2015). Measurement science roadmap for prognostics and health management for smart manufacturing systems. Roadmapping Workshop on Measurement Science for Prognostics and Health Management of Smart Manufacturing Systems. November 19-20, 2014, Gaithersburg, MD.

Roland, H.E., \& Moriarty, B. (1990). System Safety Engineering and Management, $2^{\text {nd }}$ ed. John Wiley \& Sons, New York.

Vykydal, D., Plura, J., Halfarová, P., \& Klaput, P. (2015). Advanced approaches to failure mode and effect analysis (FMEA) applications. Metalurgija, vol. 54 (4), pp. 675-678. 


\section{Appendices}

\section{Appendix A. Risk in Manufacturing Processes Survey}

1. Background
a. What is your name?
i. Is it ok if I call you by [first name]?
b. How many years have you been working with [company name]?
c. What is your current position?
d. How many years have you been working in this particular position, workstation, or job?
e. What are your everyday duties?

2. Experience

a. How would you rate your level of expertise in this position?
0 . No Experience
1. Basic Knowledge
2. Novice
3. Intermediate
4. Advanced
5. Expert

b. Can you describe your training (how many hours)?
i. Classes
ii. Education
iii. Shadowing/Apprenticeship
iv. On the job

c. Do you hold any certifications?

i. What would you consider your specialty?

d. How often do you repeat training?

3. General Work Information

a. How many hours/week do you work at this station?

i. At other stations?

b. Can you describe what goes on at this station?

c. Can you tell me what it's like on a typical good day?

d. Can you tell me what it's like on a bad day?

e. Are you given daily goals or targets?

i. Types:

1. Quality

2. Production output

3. Waste

4. Safety

ii. Who sets these targets (you, manager, company)?

f. Do you track any numbers or data on a regular basis? 
4. Maintenance

a. Can you describe what you do to keep this machine/workstation running smoothly?

i. Daily basis

ii. Weekly basis

iii. Monthly basis

b. What challenges do you face to keep this machine/workstation operational?

c. When was the last time this machine/system had to undergo maintenance?

i. What was wrong?

d. How often does this machine/system undergo maintenance?

e. How involved are you in the maintenance process?

5. Risks

a. Can you tell me about the last time you had a challenge with this machine or workstation?

i. When did that happen?

ii. How did you handle it?

b. What other challenges have you encountered with this particular machine or workstation?

i. When was the last time they happened?

ii. How were they handled?

iii. What were the end results?

iv. Did anything change as a result?

v. How frequently does this happen?

c. Has this machine or workstation ever been inactive?

i. What was the problem?

ii. How many days/hours was it inactive?

iii. Was this preventable, and if so how?

d. What can go wrong with a new job that you worry about?

e. How do you classify problems or issues (faults, errors, risks)?

f. How do you report problems or issues?

g. Is work ever rejected?

i. For what reasons?

6. Redundancy

a. Is there a backup or redundant machine/system?

i. How does it compare to this active machine/system?

b. How many other people are qualified to perform this job?

i. How does their level of expertise compare to yours?

c. Are you cross-trained to perform other jobs?

d. Do you ever work other jobs or just this one? 
7. Improvements

a. What are the bottleneck systems or processes?

b. What changes would you like to see implemented?

c. What would make your job easier?

d. Was your machine/workstation easy to learn?

e. Is your machine/workstation easy to operate?

i. Controls?

ii. Settings?

f. Does the machine/workstation give you enough feedback and information?

i. Production rate?

ii. Quality?

iii. Safety hazards?

iv. Upcoming maintenance?

v. Do you have any other ideas for improving this process? 


\section{Appendix B. Food Company - Full List of Risk Scenarios}

\section{Storing Product Failure}

a. Environment Failure

i. Factory floor becomes contaminated

ii. Airborne bacteria contaminates product

iii. Moisture contaminates product

b. Humans contaminate or tamper with product

c. Contaminant electrical controls fail/malfunction

\section{Transporting Product Failure}

a. Front End Loader Failure

i. Front end loader fails to lift the product

ii. Front end loader fails to move the product into the floor grates

b. Screw Conveyors Failure

i. Screw conveyor fails to move product to the vertical conveyor

\section{Bagging Product Failure}

ii. Screw conveyor fails to move product to the storage tank

a. Human Operator Failure

i. Human fails to get an empty bag

ii. Human fails to fill the bag with product

b. Bagging Machine Failure

i. Bagging machine fails to grip the bag

ii. Bagging machine fails to lock the bag

iii. Bagging machine fails to sense the weight

iv. Bagging machine fails to unlock the bag

c. Storage Tank Failure

i. Storage tank fails to drop the product into the bag

ii. Storage tank fails to close the hatch after dispensing the product

\section{Sealing Bags Failure}

a. Human Operator Failure

i. Human fails to orient the bag correctly

ii. Human fails to monitor the bag while being sealed

b. Sealing Machine Failure

i. Sealing machine fails to sense the new bag

ii. Sealing machine fails to grip the bag

iii. Sealing machine fails to heat seal the bag

iv. Sealing machine fails to transport the bag

v. Sealing machine fails to lay the bag flat

\section{Transporting Bags Failure}

a. Human Supervisor Failure

i. Supervisor mishandles the controls

ii. Supervisor fails to fix an unaligned bag

b. Automated Conveyor Failure

i. Conveyor fails to sense a bag

ii. Conveyor fails to move a bag

iii. Conveyor fails to properly delay a bag 


\section{Flattening Bags Failure}

a. Human Supervisor Failure

i. Supervisor mishandles the controls

b. Flattening Machine Failure

i. Flattening machine fails to sense a bag

ii. Flattening machine fails to flatten a bag

iii. Flattening machine fails to properly move a bag

\section{Palletizing Bags Failure}

a. Forklift Failure

i. Forklift fails to move an empty pallet

b. Human Supervisor Failure

i. Supervisor fails to properly adjust the settings

ii. Supervisor fails to start/stop the process

iii. Supervisor fails to fix a fallen bag

c. Palletizing Robot Failure

i. Palletizing robot fails to sense a bag

ii. Palletizing robot fails to grip a bag

iii. Palletizing robot fails to lift a bag

iv. Palletizing robot fails to position a bag

v. Palletizing robot fails to place a bag on the pallet

\section{Final Preparation Failure}

a. Forklift Failure

i. Forklift fails to lift a full pallet

ii. Forklift fails to transport the pallet to the packager

b. Pallet Packager Failure

i. Packager fails to rotate the pallet

ii. Packager fails to dispense shrink wrap

c. Human Operator Failure

i. Operator fails to operate the machinery

ii. Operator fails to transport the pallet to the delivery area 


\section{Appendix C. Tool Company - Full List of Risk Scenarios}

\section{Production Failure}

a. Cutoff Saw Failure

i. Operator failure

ii. Raw material is too heavy for the forklift

iii. Machine failure

iv. Cutting blade needs replacing

v. Parameters are incorrect

vi. Health of machine degrades

vii. Coolant/oil needs replacing

b. Heat Treatment Failure

i. Operator failure

ii. Material failure

iii. Machine failure

iv. Parameters failure

v. Health of machine degrades

vi. Sensors failure

c. Lathe Failure

i. Operator failure

ii. Material failure

iii. Machine failure

1. Overheating

2. Bar feeder out of alignment

3. Machine part breaks

iv. Program failure

v. Cutting blade/insert breaks

vi. Incorrect tool selected

vii. Health of machine degrades

viii. Coolant/oil needs replacing

d. Grinder Failure

i. Operator failure

ii. Material/product breaks or deforms

iii. Machine failure

1. Collates wear out

2. Belts wear out

iv. Cutting blade wears out

v. Parameters are incorrect

1. Wrong collates used

vi. Health of machine degrades

vii. Coolant/oil/collates need replacing

e. 5-Axis Failure

i. Operator failure

1. Wrong input

2. Wrong program

ii. Material failure - part breaks in machine 
iii. Machine failure

1. Overheating

2. Spindle breaks

3. Table becomes out of alignment

4. Fixtures wear out

iv. Program failure

1. Program is incorrect

v. Cutting blade/insert failure

vi. Tool failure

1. Wrong tool selected

2. Wrong tool carousel selected

3. Tool length incorrect

vii. Sensors failure

1. Sensors become blocked with coolant

2. Gantry blowers fail to clear sensors

viii. Parameters failure

ix. Health/condition failure

x. Coolant/oil needs replacing

f. Black Oxide Failure

i. Operator failure

ii. Material failure

iii. Machine failure

iv. Parameter failure

v. Condition failure

vi. Sensors failure

\section{Engineering Failure}

a. Computer Failure

i. Hardware failure

ii. Software failure

b. Program Failure

i. Bad program (requires modification by the operator)

c. Engineer Failure

i. Engineer incident

ii. Engineer injury

iii. Engineer absent

d. Schedule Failure

i. Not available for support (during off-shifts)

\section{Maintenance Failure}

a. Internal Network Failure

i. Network crashes

b. Maintenance Crew Failure

i. Incident

ii. Injury

iii. Absent 
c. Engineer Failure

i. Bad solution to new maintenance issue

d. Inventory Failure

i. Machine part

ii. Cutting insert

iii. Coolant

iv. Oil

\section{Logistics Failure}

a. Staff Failure

v. Not enough support

b. Inventory Failure

vi. Raw materials

vii. Tools

viii. Machine parts

ix. Cutting inserts

x. Coolant

xi. Oil

c. Order Failure

d. Coordination Failure

xii. Departments

xiii. Customer

e. Schedule Failure

5. Management Failure

a. Manager Failure

b. Supervisor Failure

c. Order Failure

i. Bill of materials

ii. Schedule

iii. Identification

d. Policy Failure

e. Schedule Failure

6. Environment, Health, and Safety Failure

a. Staff Failure

b. Incident

iv. LTI

v. DART

vi. Injury/Illness

vii. Near Miss

viii. Hazard

c. Investigation Failure

d. Standards Failure

e. Policy Failure

f. Schedule Failure 


\section{Quality Failure}
a. Staff Failure
b. Standard Failure
c. Schedule Failure
d. Labeling Failure
e. Packaging Failure 
Appendix D. Support Flowcharts for RFRM Phase 5 Multi-Criteria Evaluation

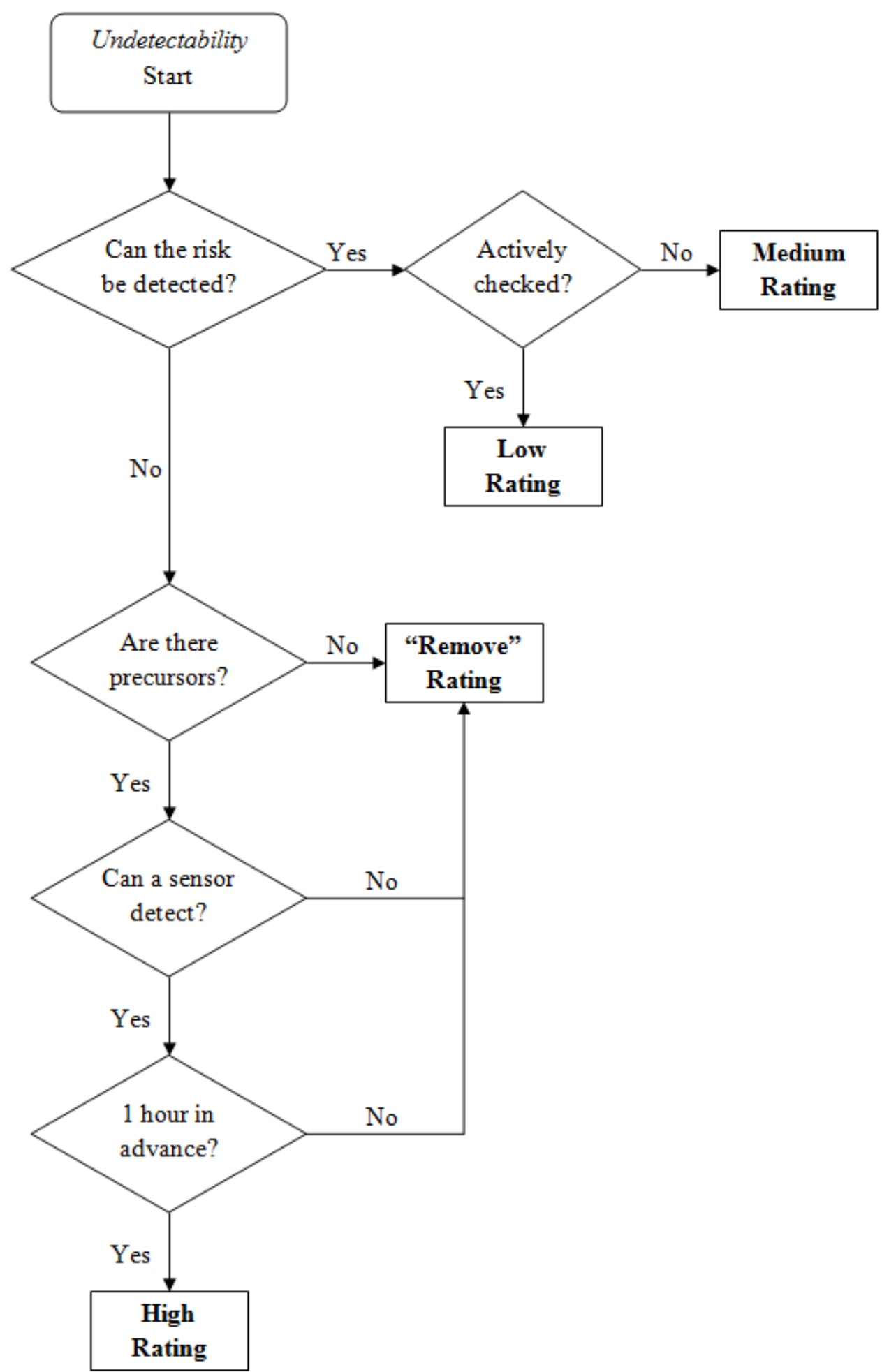




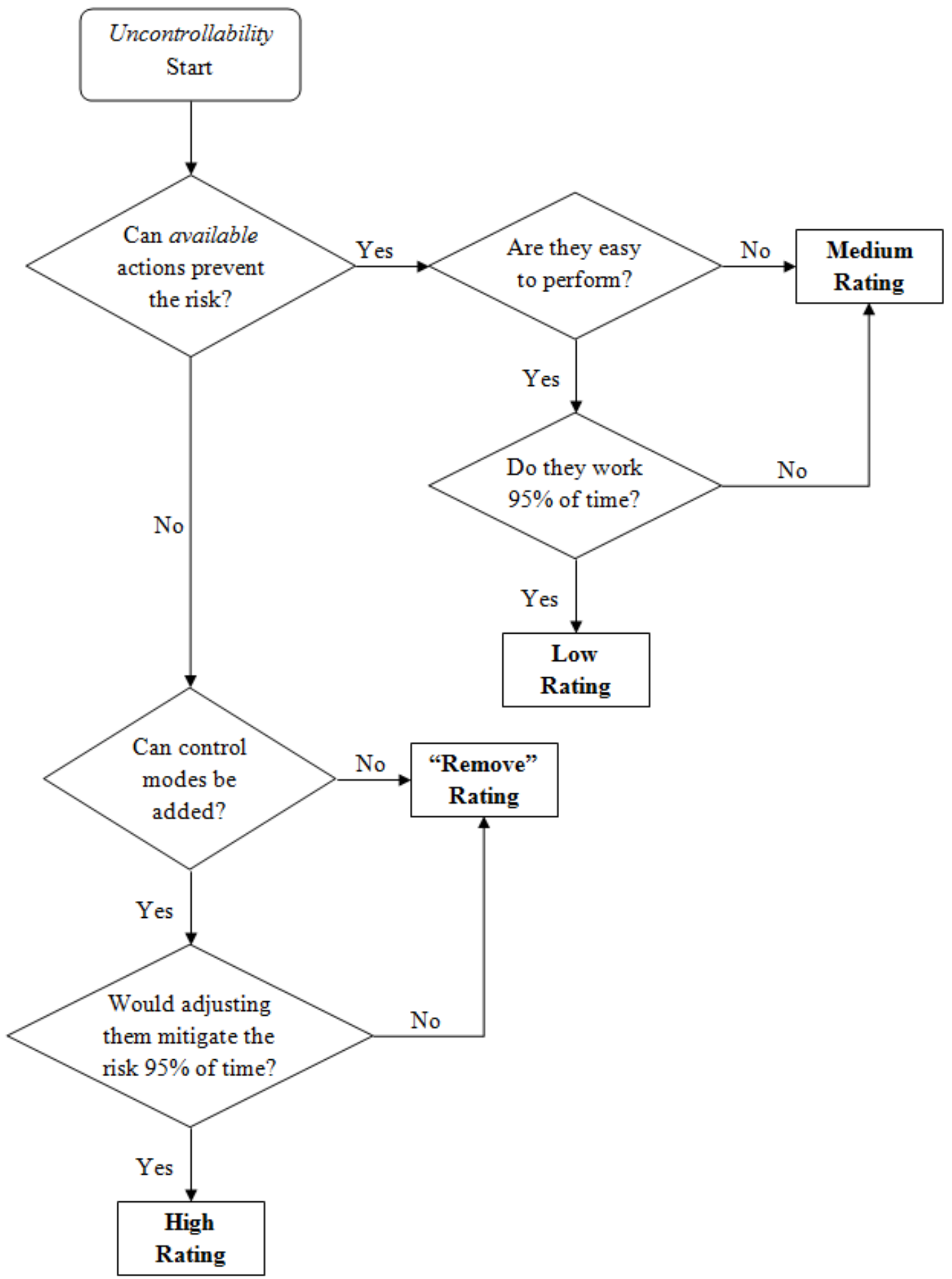



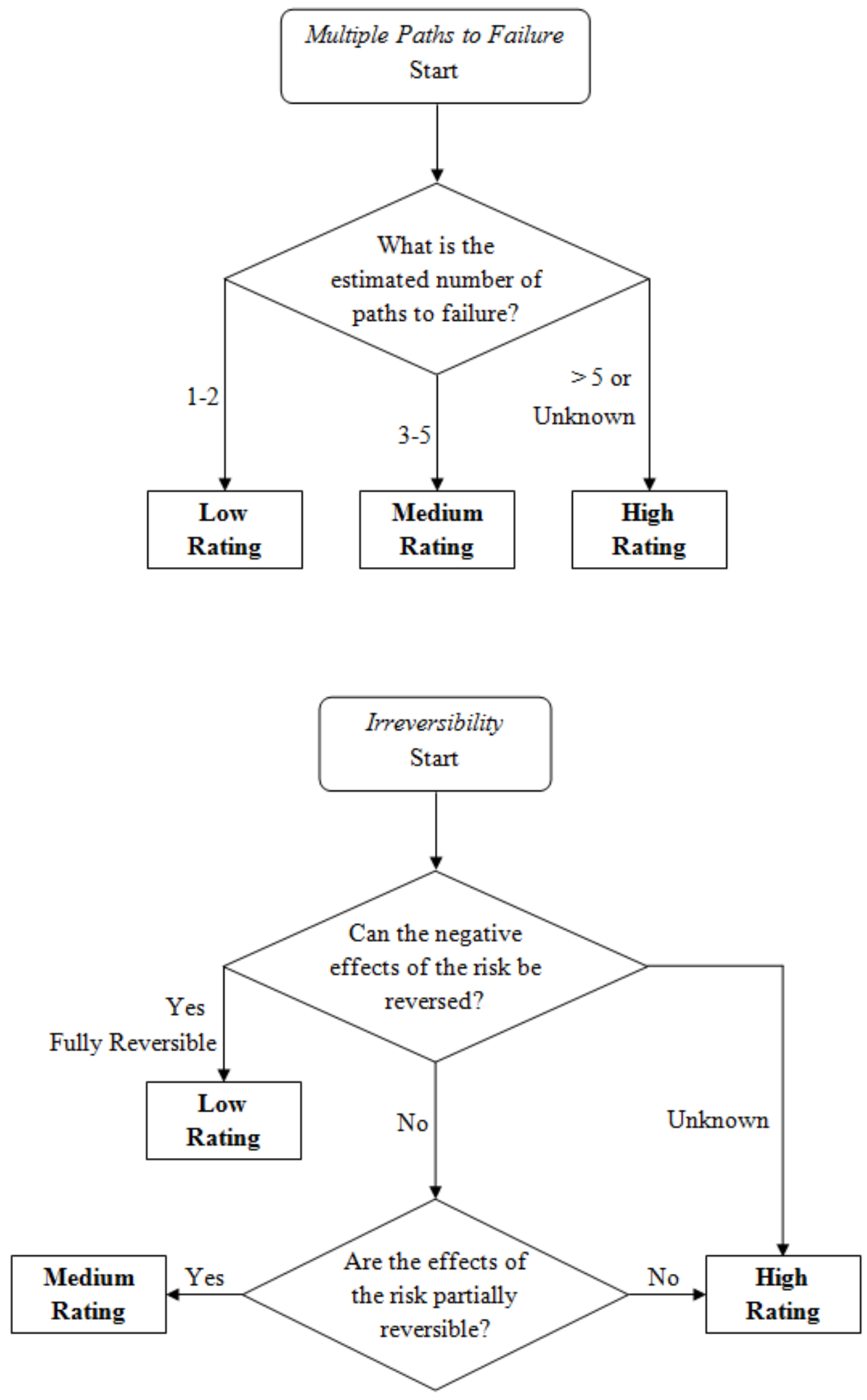

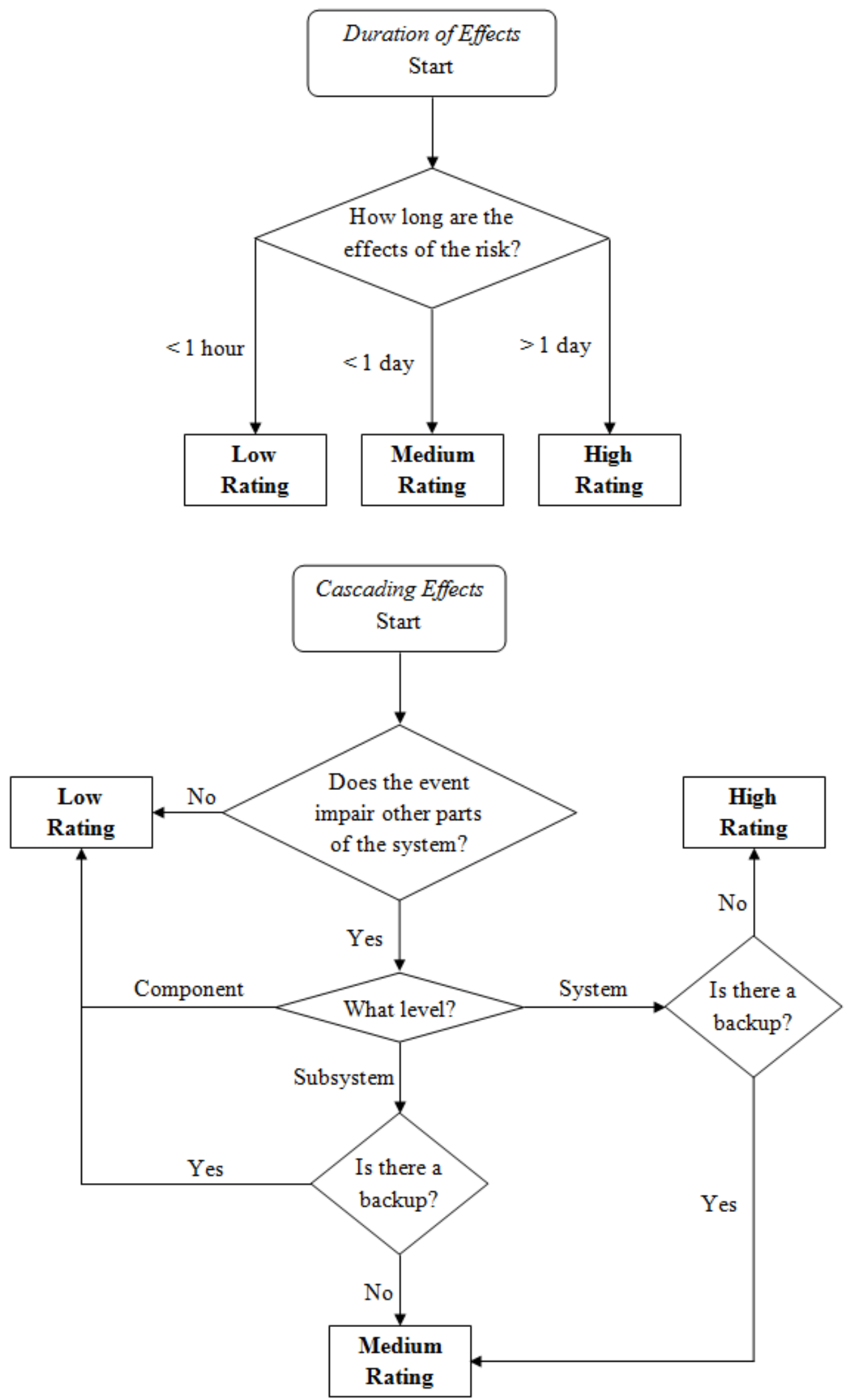


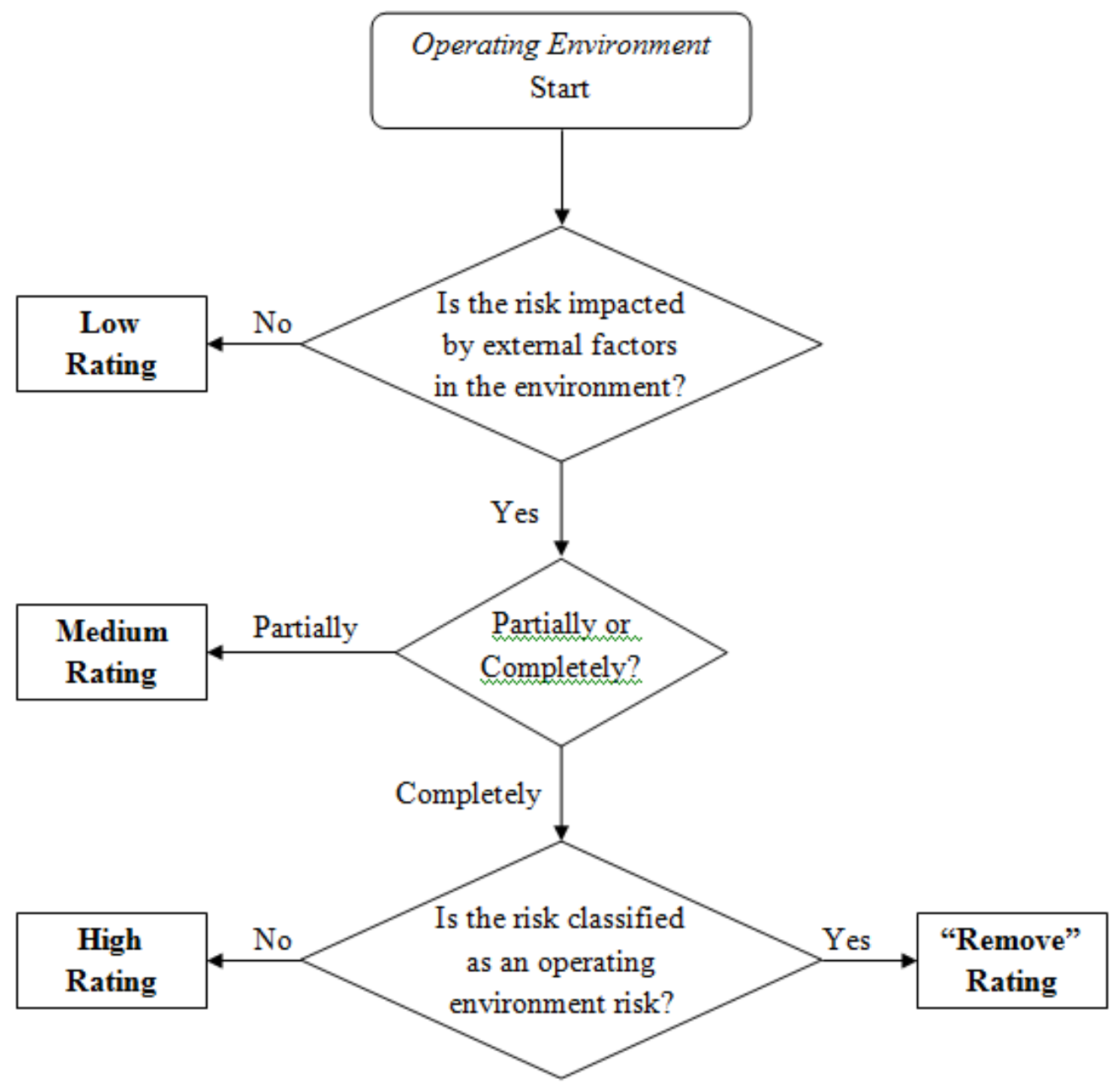



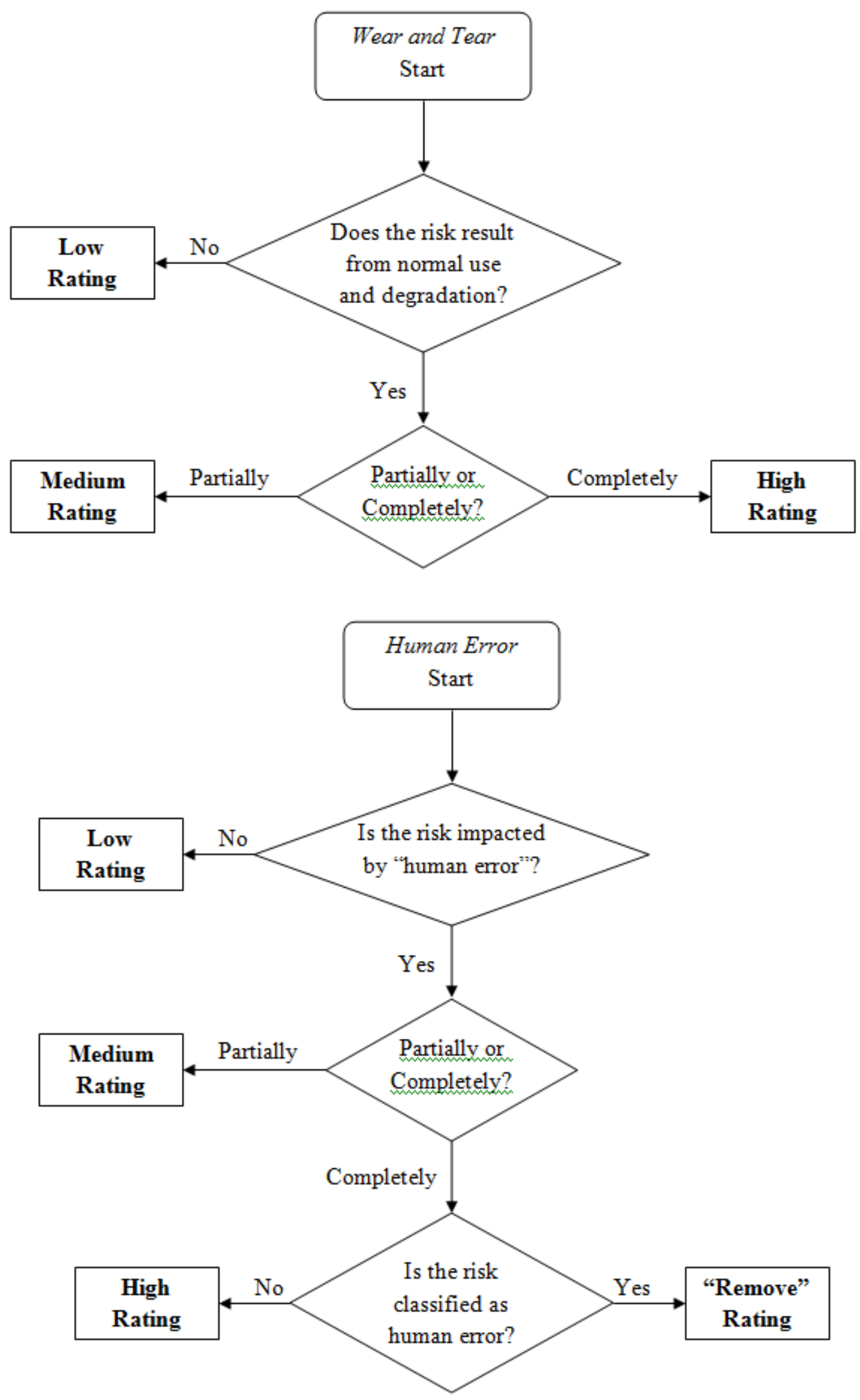


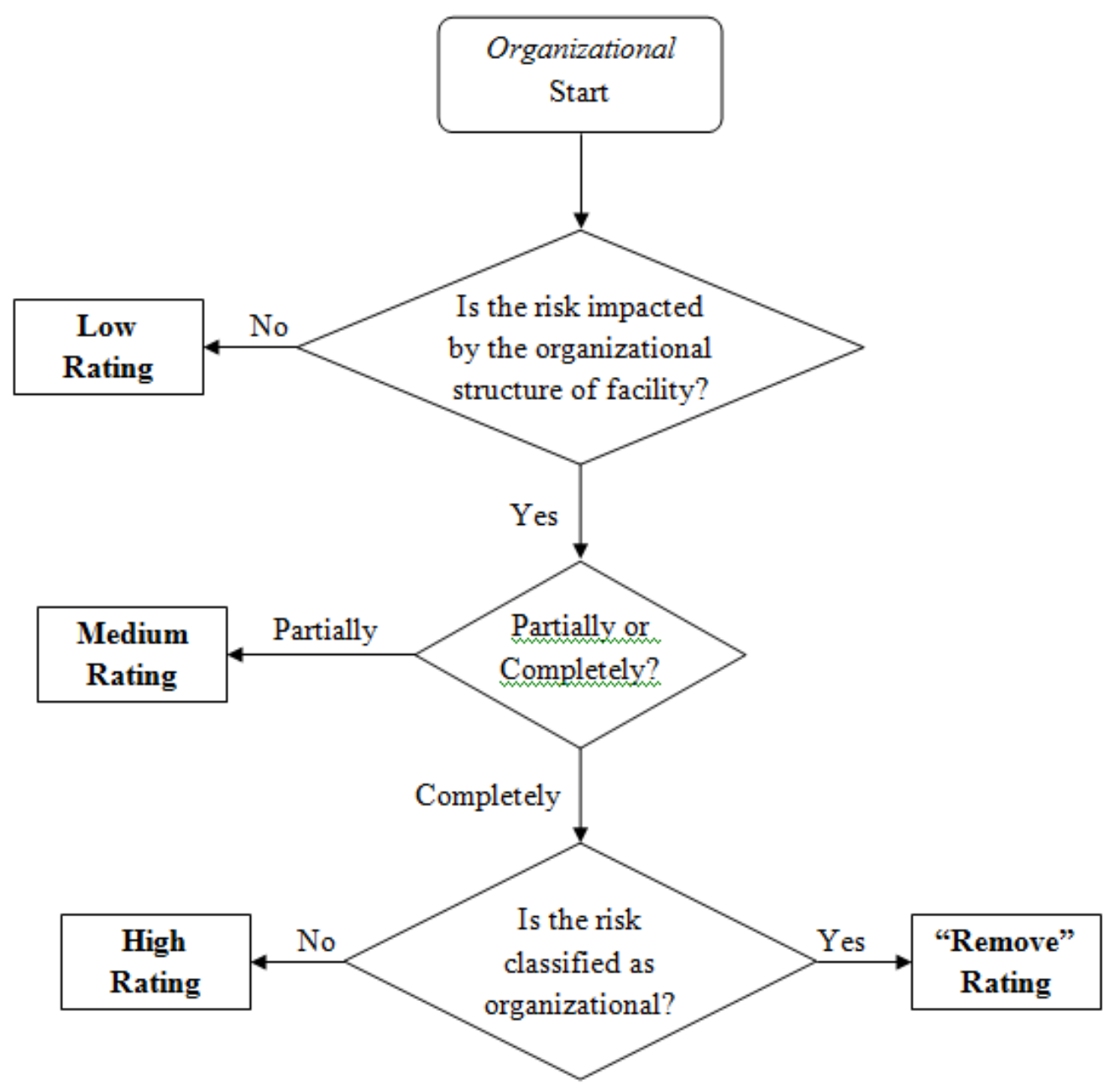



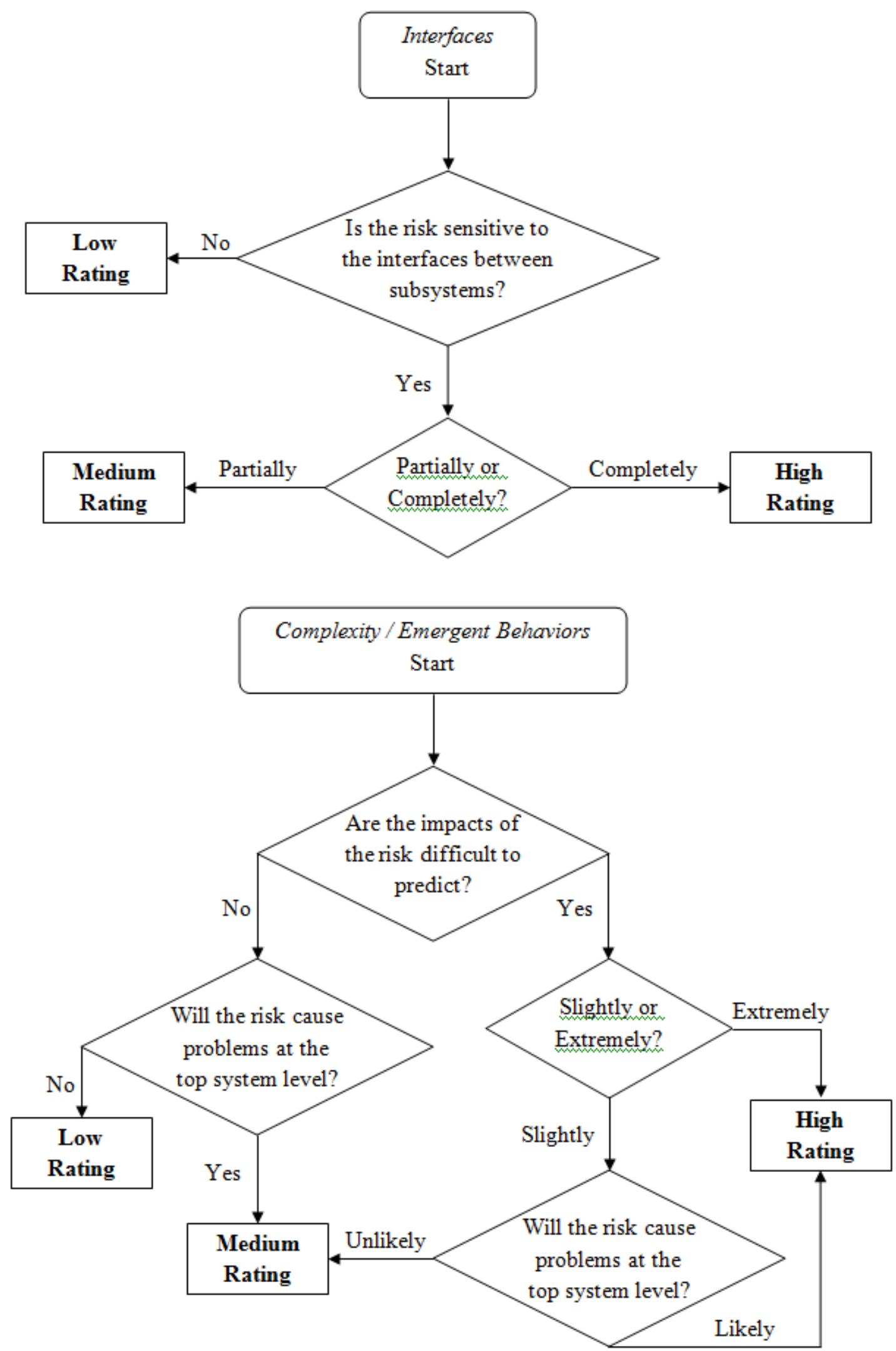


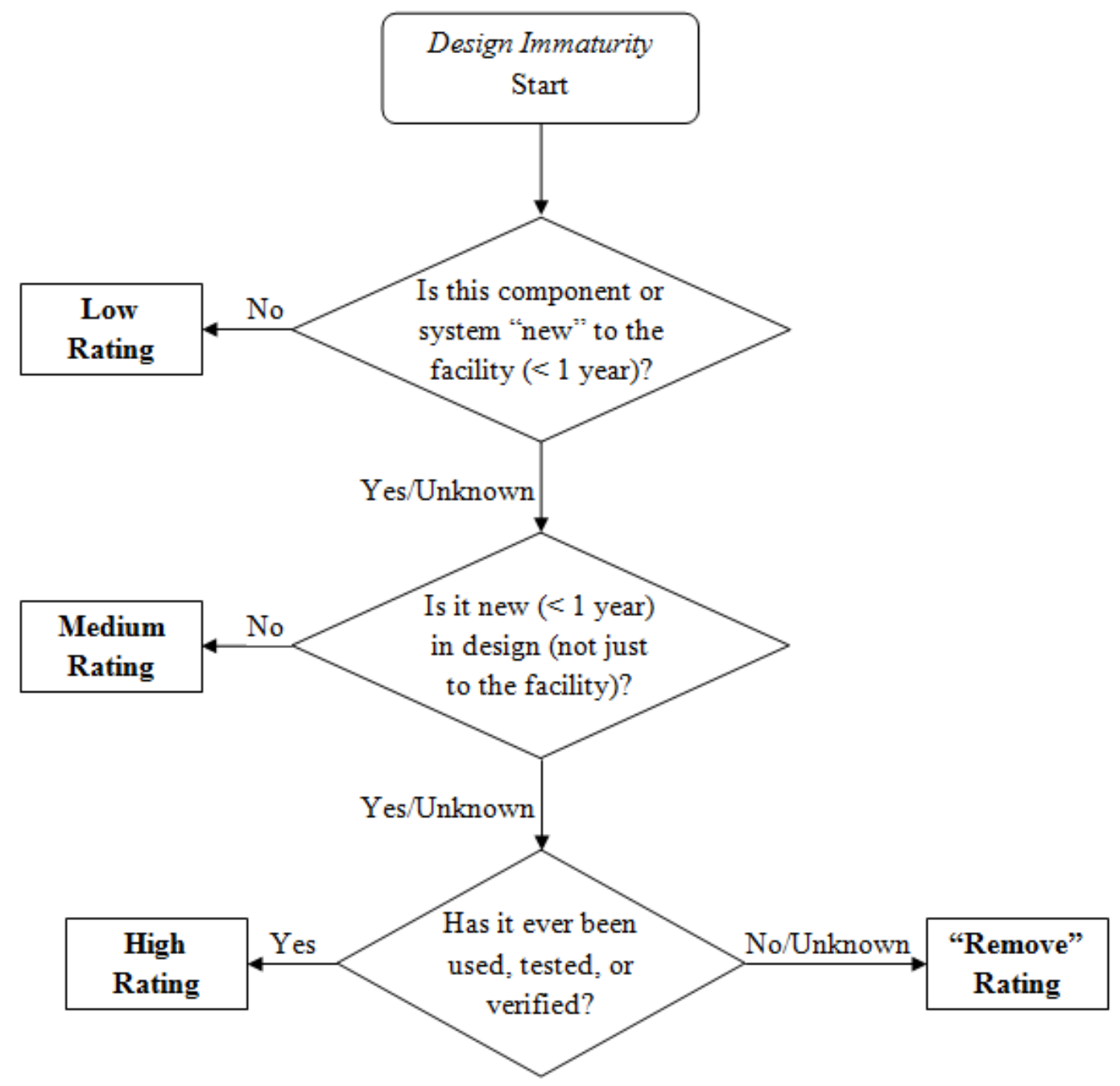

